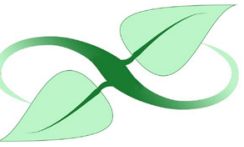

Check for updates

\title{
Canada and the Changing Global NHP Landscape: The 17th Annual Conference of the Natural Health Products Research Society of Canada
}

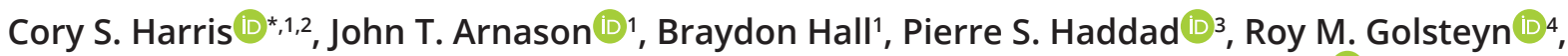
Bob Chapman', Michael J. Smith ${ }^{6}$, Sharan Sidhu7, Pamela Ovadje ${ }^{8}$, Halton Quach ${ }^{7}$, Jeremy Y. Ng (10,11

${ }^{1}$ Department of Biology, University of Ottawa, Ottawa, ON, Canada ${ }^{2}$ Department of Chemistry and Biomolecular Sciences, University of Ottawa, Ottawa, ON, Canada ${ }^{3}$ Department of Pharmacology and Physiology, University of Montreal, Montreal, QC, Canada ${ }^{4}$ Department of Biological Sciences, University of Lethbridge, Lethbridge, AB, Canada ${ }^{5}$ Dosecann Inc., Charlottetown, PE, Canada ${ }^{6}$ Michael J Smith and Assoc, Stratford, ON, Canada ${ }^{7}$ Numinus Wellness Inc., Vancouver, $B C$, Canada ${ }^{8}$ Evexla Bioscience Consulting, Calgary, $A B$, Canada ${ }^{9}$ Department of Biology, York University, ON, Canada

${ }^{10}$ Department of Health Research Methods, Evidence and Impact, McMaster University, Hamilton, ON, Canada ${ }^{11} \mathrm{NHP}$ Publications, Toronto, ON, Canada *@ charris@uottawa.ca

\begin{abstract}
The 17th Annual Natural Health Products Research Conference hosted by the NHP Research Society of Canada (NHPRS) will be held from June 7-9 \& 14-16, 2021, virtually hosted by the University of Ottawa, in Ottawa, Ontario. Founded in 2003 by a collaboration of academic, industry, and government researchers from across Canada, the NHPRS is a Canadian federally incorporated non-profit organization. The goals of the NHPRS are; (a) to promote scientifically rigorous research and education on natural health products (NHPs), (b) to develop a national research community that encompasses academic, health professional, government and a broad-base of industry stakeholders, (c) to support national research priorities that best enable the informed and appropriate use of NHPs that are safe and efficacious, (d) to increase the capacity for NHP research and education, (e) to facilitate effective NHP knowledge transfer and translation, $(f)$ to support the use of science-based product quality standards and the use of well-characterized materials and protocols in research and (g) to foster value-chain development through interdisciplinary NHP research collaborations and networking. To forward these objectives, the NHPRS has held annual research conferences since inception with themes that highlight the important trends in the ever growing and changing field of NHP Research. The theme of the 17th Annual Conference is "Canada and the Changing Global NHP Landscape", featuring six "live" sessions (Global Stewardship and Sustainability, NHPs in the Time of the Pandemic, Indigenous Traditional Medicine, Global Advances in NHP Research, NHPs in Clinical Practice and Research, and Regulation, Policy, Commerce) and contributed sessions on topics such as cancer, cannabis, functional foods. natural product chemistry, and more. The abstracts are ordered alphabetically by presenting author last name, divided by topic sessions. For more information, please visit: http://www.nhprs.ca/.
\end{abstract}

KEYWORDS: NHP Research Society of Canada, NHPRS, natural health products, research 


\section{Acknowledgements}

On behalf of the NHP Research Society of Canada we gratefully acknowledge the support of our conference sponsors and partners, and the contributions of the Program Committee, Local Organizing Committee, NHPRS Board of Directors, student interns and volunteers. We would like to acknowledge the University of Ottawa Conferences team led by Sanae Elamrani and IT support team led by Pierre Tardif.

Note: Corrections to a few abstract author names were made after the original version was published on June 06,2021 , however, all content remains unchanged. We regret any inconvenience caused.

The NHPRS thanks the sponsors and partners of the 2021 Annual Conference for their support!

\section{Platinum-Level Sponsor}

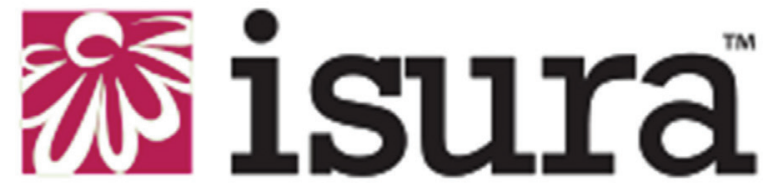

\section{Bronze-Level Sponsors}

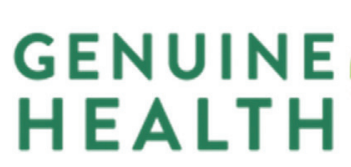

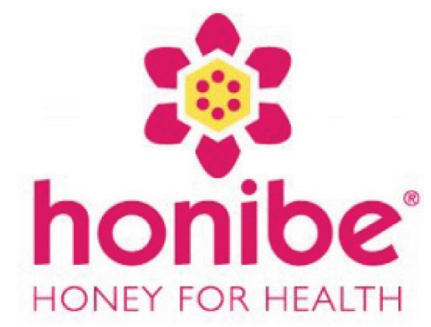

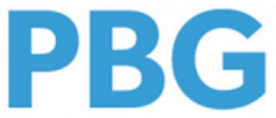

BIOPHARMA SCANME
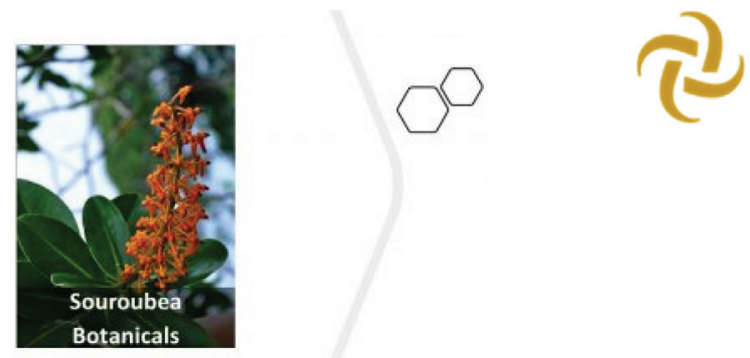

PRINCE EDWARD ISLAND

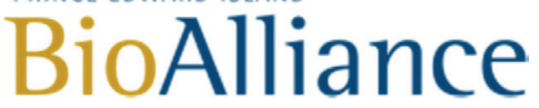

Partners of the 2021 NHPRS Annual Conference
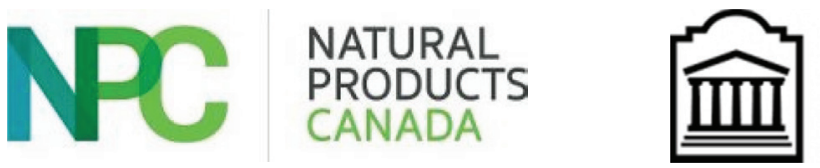

uOttawa 


\section{Table of Contents}

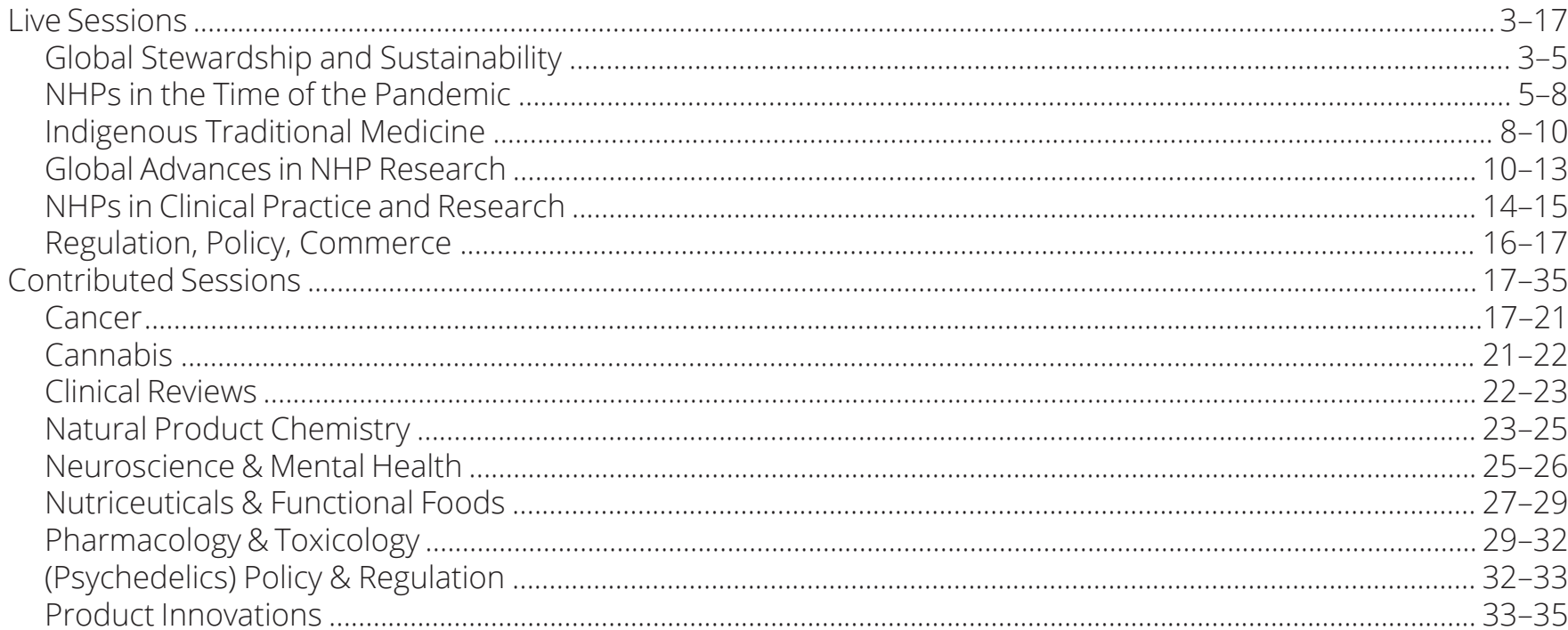

\section{Live Sessions}

\section{Global Stewardship and Sustainability}

\section{Responsible Sourcing in the Botanical Industry \\ Ann Armbrecht ${ }^{1}$ \\ 'Sustainable Herbs Program, USA}

The Sustainable Herbs Program of the American Botanical Council is following medicinal plants from their point of origin through their value networks and supply chains to raise industry and consumer awareness about issues of sustainability, regeneration, and ethical practices. In this presentation, Ann Armbrecht will discuss best practices around responsible sourcing and sustainability in the natural products industry and discuss why these practices are crucial to the long-term success of the industry.

\section{Natural Product Sustainability Approach: Industrial Insight Antoine Bily' \\ 'Gauvidan, France}

Givaudan is a global leader in flavours and natural ingredients for health and wellness. In our journey to become climate positive, our research activities look at both how to reduce emissions, increase process yield and carbon storage along our supply chain. This presentation focuses on research activities in greener extraction practices including use of solar energy and innovative technologies to intensify extraction of natural raw materials, alternative solvents including natural deep eutectic solvents, biorefinery-upcycling and use of modelization and life cycle assessment in new process development.

\section{Soil Health, Climate \& Supplements \\ Bethany Davis ${ }^{1}$ \\ ${ }^{1}$ MegaFood, USA}

Bethany Davis will discuss the role that natural product companies play in the global soil health crisis. Nutrient levels in food have been declining, atmospheric greenhouse gas (GHG) has been rising and the health of topsoil globally is on life support. As it turns out, all of these issues are correlated and Natural Health Products that contain agricultural materials have an important role to play. Natural Health Products are meant to be safe, effective and of high quality. But what about the impact of the supply chain and production process of these products? I will review the nutritional impact of industrial agriculture and outline how regenerative practices have the potential to support soil health, 
reduce GHG, result in more nutrient dense foods and herbs, positively impact water quality and the water cycle all while supporting biodiversity. We will cover research, practices, measurement tools, capabilities and how companies can move from extractive supply chains to truly regenerative ones. The win-for-all solution is to have more sustainable standards \& practices which results in healthier populations and healthier soil.

\section{Introduction of Ma'afala Breadfruit Flour to World Commercial Markets}

\section{Abisola Kehinde', Lauren A. E. Erland', Diane Ragone ${ }^{2}$, Susan J. Murch ${ }^{1}$}

${ }^{1}$ Department of Chemistry, University of British Columbia, Kelowna, British Columbia, Canada, V1V 1V7

${ }^{2}$ Breadfruit Institute, National Tropical Botanical Garden, Kauai, Hawaii, USA

Breadfruit (Artocarpus altilis) is an underutilized staple crop originating from the Pacific Islands. Beginning in 2008, worldwide distribution of breadfruit trees for food security has planted more than 150,000 trees in $>50$ countries. Chefs at elite restaurants are now including breadfruit in their menus and a distillery has opened that produces vodka from breadfruit. Hundreds of products are entering the market. The first commercial cultivar, Maafala, originated in Samoa and is one of the first crops distributed under a Memorandum of Understanding that returns a portion of all profits to the country of origin for education, conservation and sustainability projects. Facilities have been built in Costa Rica, Hawaii, Samoa, Vietnam and other places to harvest and process Ma'afala fruits into a gluten-free flour. Gluten-free Ma'afala flour is now available in Canadian grocery stores and through international delivery services such as Amazon. Ma'afala flour contains high-quality protein with essential amino acids in better ratios than wheat, corn, rice, soybean, yellow pea, and other plant proteins. The flour is also more digestible than wheat flour, has high-energy value and did not induce gut inflammatory responses in animal models. Our objective was to discover whether Maafala fruit grown in different locations and under different conditions has consistent quality characteristics. We analyzed fruit size, edible yield, starch, and flour characteristics from 86 commercial Maafala clones planted at 6 locations in Hawaii and compared the results to the original source tree in Maui. Locations were selected to represent the wet and dry sides of the islands, irrigated and non-irrigated trees, and different agricultural management practices. Our data show that fruit size, edible yield, starch yield, starch-bound protein content, and flour protein are significantly increased by irrigation and/or rainfall. The studies provide basic data to support agronomic practices in breadfruit growing regions.

\section{Evolving from Plant to Product-Respecting the Process Connie Kehler ${ }^{1}$ \\ ${ }^{1}$ Executive Director, Canadian Herb, Specialty Agriculture and Natural Health Product Coalition, Canada}

Ensuring your raw material supply is not only sustainable but that its origins and traditions respected and supported good environmental practices is a daunting task that requires communication and collaboration between all players in the supply chain. This is particularly important to researchers and formulators to ensure research integrity that is maintained, repeatable and measurable while still respecting sustainability and effective stewardship. In a world where the next "best thing" often becomes a rush for supply in a very short window, problems almost always arise. The choice becomes commodity type supply with unknown or unconfirmed sourcing or doing the work to build relationships and dialogue with the supply source, including a realistic understanding about what is possible and what is not. What is an ethical supply chain-is it important to understand how to get source material that is sustainable, respects the people, the plants and the place, and is still viable for quality end product? This talk will walk you through the steps already in place, identify potential problems along the way, and use examples to illustrate possible solutions.

\section{Surveying Natural Health Product Researchers' Attitudes towards Publishing and a New Field-Specific Journal}

Jeremy Y. Ng${ }^{1}$, Halton Quach ${ }^{1}$, Fred Ashbury ${ }^{2}$, Kieran Cooley ${ }^{3}$, Pierre S. Haddad ${ }^{4}$

${ }^{1}$ Department of Health Research Methods, Evidence, and Impact, Faculty of Health Sciences, McMaster University, Hamilton, Ontario, Canada

2Institute of Health Policy, Management and Evaluation, University of Toronto, Toronto, Ontario, Canada

${ }^{3}$ Department of Research, Canadian College of Naturopathic Medicine, Toronto, Ontario, Canada

${ }^{4}$ Department of Pharmacology and Physiology, Faculty of Medicine, University of Montreal, Montreal, Quebec, Canada

This project aims to understand the attitudes and opinions of natural health product (NHP) researchers towards open access publishing and a new field-specific journal. Following the completion of an NHP researcher database (consisting 
of NHP researchers from the regions of Canada, United States, West Europe and Australia), a descriptive cross-sectional survey was developed and NHP researchersidentified through the aforementioned database were invited to participate. An active, snowball sampling method was used to recruit participants which consisted of international English-speaking NHP researchers. Participants were permitted to skip questions at their discretion. A total of $167 \mathrm{NHP}$ researchers participated, the majority of whom had research interests/publication histories relating to the traditional use, phytochemical composition, and therapeutic properties of NHPs ( $\mathrm{n}=102 / 138,73.9 \%)$, or NHPs in clinical settings and healthcare education (n $=61 / 138,44.2 \%)$. The majority of participants expressed being "very familiar" with the concept of an open access journal $(\mathrm{n}=108 / 139,77.7 \%)$, and had published in them $(\mathrm{n}=110 / 139,79.1 \%)$. Sixty-two respondents $(44 \%)$ expressed no preference towards publishing their research in open access journals compared to subscription-based journals whereas 35.3\% $(n=49 / 139)$ of respondents expressed interest in publishing in the former and 20.1\% $(n=28 / 139)$ in thelatter. Impact factor $(\mathrm{n}=107 / 139,77.0 \%)$ and journal reputation $(\mathrm{n}=105 / 139,75.5 \%)$ were perceived to be the two most important elements considered when selecting a journal to submit a manuscript. The majority of participants saw benefit in a new field-specific journal that would benefit the NHP research community $(n=110 / 122,90.2 \%)$. The findings of this study indicate that the majority of NHP researchers surveyed are familiar with open access publishing, view a new field-specific journal as beneficial and have an interest in publishing their research in it. Ensuring that the journal remains affordable, publishes high-quality research, and maintains a broad scope can maximize the publication's long-term success.

\section{Four-Year Field Performance of Open-Pollinated Rhodiola Rosea Family Tests at Two New Brunswick Locations}

\section{Ron Smith ${ }^{1}$, Stewart Cameron ${ }^{1}$}

${ }^{1}$ University of New Brunswick, Canada

Rhodiola rosea roots are an important adaptogenic medicinal material in short supply globally, for which additional sources are needed to fill increasing demand. Root biomass production and bioactive component profiles (rosavins and salidroside) from 42 open-pollinated seedlots from wild plants collected in New Brunswick and Nova Scotia were assessed at two field sites. Six additional seedlots from partners in Alberta and Quebec were obtained and tested. Seedlings of two commercially grown genotypes from the Alberta Rhodiola Rosea Growers Association (ARRGO) were also planted to obtain a preliminary evaluation of their growth potential in New Brunswick.

Significant variations in growth and chemical content were found both among and within families. After four years in the field, there was a 15-fold difference among families in total root dry weight, and a 30-fold difference between the best and the worst individuals. There was more than a 10 -fold difference among families in the mean content of the different rosavins and salidroside.

Mean total rosavin content of the top performing families met or exceeded published results for commercially produced plants of the same age and with the yields reported from commercial plantations at ARRGO.

Although a general positive correlation existed between plant growth (biomass) and levels of rosavins, growth and chemical content among seedlots varied somewhat independently. Increasing crop yields through selecting phenotypes superior in both root biomass production and phytochemical content, in combination with improved cultivation practices are discussed. The high degree of variation in both growth and phytochemical yield would be highly advantageous for commercial production and crop improvement for Rhodiola in New Brunswick.

Study results indicate that Rhodiola rosea represents a substantial opportunity as a new, high-value crop for Atlantic Canadian farmers. The opportunity requires a long-term investment, but one that can be demonstrated to be economically viable and highly profitable.

\section{NHPs in the Time of the Pandemic}

\section{Caring for COVID-19: a Herbalist's Approach Caroline Gagnon ${ }^{1}$ \\ ${ }^{1}$ Herboriste-Thérapeute Accréditée, Directrice de FloraMedicina, Canada}

In March 2020, the COVID-19 pandemic surprised the world with the diversity of symptoms associated with the disease and the difficulty of treating those suffering from it. In hospitals in some countries more accustomed to integrative medicine, notably in China, protocols based on medicinal plants were put in place to treat people affected by 
SARS-CoV-2. In the Western world also, herbalists have used their pharmacopoeias to try to alleviate the symptoms of the disease. Rapidly, from observations and clinical experiences, practitioners from different countries shared their protocols to make accessible a knowledge that is evolving daily, keeping up with the growing medical knowledge on this pathology. By studying the pathogenesis of the virus, herbalists were able to develop safe and effective herbal protocols tailored to the symptoms of SARS-CoV-2. They were able to draw on, from their pharmacopoeias, those plants whose specific medicinal properties could respond to the symptoms observed, following three main axes. 1Some antiviral plants have demonstrated an ability to prevent the S protein from connecting to the ACE2 receptor, thus decreasing the intracellular replication of the virus. 2- Other plants have demonstrated an ability to modulate the immune response to prevent the surge of the immune reaction causing the cytokine storm; 3- The observation of endothelial damage and clot formation induced by COVID-19 has led herbalists to add vascular protective and antithrombotic herbs to their protocols.

Complying with public health guidelines, without claiming to cure COVID-19 and without positioning themselves as an alternative to conventional medical care, herbalists used plants to support the body's different systems and functions in order to help it regain an optimal state of health after the infection. With the health care system already overwhelmed by patients with severe forms of the disease and respiratory distress, herbalists were able to accompany people with mild forms of the disease and those struggling with the long form but not receiving medical care.

By explaining the particularities of the herbalist approach and demonstrating the composition of a care protocol based on the experience of the speaker, this paper aims to restore the complexity of the use of medicinal plants in a care context.

\section{Herbal Medicines and Natural Products in the Context of COVID-19 \\ Michael Heinrich ${ }^{1}$ \\ ${ }^{1}$ Research Group 'Pharmacognosy and Phytotherapy', School of Pharmacy, University College London, UK}

Within our living memory, the years 2020 and 2021 have been unprecedented in terms of health care challenges, disease risks and the impact on peoples' lives. Plant (and fungi) derived products have not been high on the agenda in the public debate, but at the same time we have seen a surge in demand in what is called Natural Health Products in Canada.

Faced with this global crisis, there are four main approaches where medicinal plants may play a role-the adjuvant treatment using herbal medicines [1], the use of natural products in drug discovery [2], disease prevention and general public health measures. Silveira et al. [1] argue that a set of herbal medicines have safety margins superior to those of synthetic OTC drugs and sufficient evidence allowing a clinical discussion about their potential use as adjuvants, i.e. the symptomatic treatment of mild to potentially moderate forms of COVID-19. These will not cure or prevent the disease. Their benefits lie in improving general patient well-being and enable patients to actively engage with the treatment process (cf. also Zhuang et al. [3] on TCM). The development of directly acting antiviral agents is a longer term strategy, has been advocated in the context of drug discovery, but it remains uncertain whether directly acting anti-viral agents can be developed. Preventive strategies against viral infections, focusing both on public health measures (i.e. at a policy level) but also on personal preventive measures, have so far been explored only in a very limited way. Clearly, there is a need to rethink resource use more generally and, while this is not a topic specific to medicinal plants, there is a need for developing much more sustainable uses of the global biodiversity. The One Health approach, i.e. 'the collaborative efforts of multiple disciplines working locally, nationally, and globally, to attain optimal health for people, animals and our environment' [4], heavily relies on a broad range of preventive measures and to changes in the production systems and value chains. Natural Health products are just one of many possible foci, and certainly one which is currently underrepresented. Lastly, personal hygiene has become an important topic, including for example, the use of essential oils and other substances to prevent the spread of the virus and the transmission of the diseases.

One of the most important lessons resulting from this is in fact an old one-this field needs to engage with a wide range of other sectors to enable solutions which are based on multidisciplinary strategies.

[1] Silveira, D., Prieto-Garcia, J.M., Boylan, F., Estrada, O., Fonseca-Bazzo, Y.M., Jamal, C.M., Magalhães, P.O., Oliveira, M., Tomczyk, M., Heinrich, M. (2020) COVID-19: Is there evidence for the use of herbal medicines as adjuvant symptomatic therapy? Front. Pharmacol. doi: 10.3389/fphar.2020.581840

[2] Hensel A, Bauer R, Heinrich M, Spiegler V, Kayser O, Hempel G, Kraft K. Challenges at the Time of COVID-19: Opportunities and innovations in antivirals from nature. Planta Med. 2020 May 20. doi: 10.1055/a-1177-4396. 
[3] Zhuang W, Fan Z, Chu Y, et al. Chinese patent medicines in the treatment of Coronavirus Disease 2019 (COVID-19) in China. Front Pharmacol. 2020;11:1066. Published 2020 Jul 17. doi:10.3389/fphar.2020.01

[4] American Veterinary Medical Association 2008 "One Health: A New Professional Imperative" 15 July 2008. American Veterinary Medical Association (AVMA), Schaumburg Il, US.

\section{Natural Health Products Research in the Time of COVID-19 Pandemic}

\section{Ossy M. J. Kasilo'}

${ }^{1}$ Regional Adviser, Traditional Medicine at the World Health Organization, Africa

The keynote address will outline the WHO policy and regulatory framework at global, continental and regional levels governing the integration of traditional and complementary medicine into national health systems and highlight WHO's efforts in supporting countries to find homegrown solutions to the COVID-19 pandemic.

These efforts include four main components: First, setting-up with the Africa Centre for Disease Control and Prevention (CDC) and African Union Commission for Social Affairs and jointly chairing, a Regional Expert Advisory Committee on Traditional Medicine (TM) for COVID-19 Response to improve standards, pool expertise for multi-centre studies, provide independent scientific advice and support countries to generate scientific evidence on the safety, efficacy and quality of TMs through research and development (R\&D). Second, development of research protocols and other technical documents for randomized clinical trials of herbal medicines for COVID-19. Phases I-III clinical trials are now underway for TM in several countries in the African Region. Preliminary results on the safety, efficacy and quality of the TMs against COVID-19 from some countries are very encouraging. Third, building countries' capacity and strengthening protocol development, preclinical and clinical research through good clinical practice and good laboratory practice respectively; implementation of national policies, standards, strengthening of regulatory systems to engage the private sector, and to build the expertise needed to produce herbal medicines (vaccines and other products) locally. These actions will facilitate integration of TM as a key part of national health systems, elevate African TM on the world stage, strengthen countries self-reliance and ensure Africa is no longer at the back of the queue for essential commodities. Fourth, awareness creation in communities about COVID-19, through operationalization of a WHO guide on the potential contribution of TM to COVID-19 response. The guide outlines the role of traditional health practitioners in the prevention and control, early recognition and referral of people infected with COVID-19 to health facilities for proper diagnosis and management especially in communities where they live.

The presentation will underscore the potential socio-economic development benefits of herbal medicines for COVID-19. Finally, it will outline the challenges countries are facing in conducting R\&D during COVID-19 pandemic and the way forward in addressing some of the challenges.

\section{Natural Health Products: Clinical Outcomes in Active COVID-19}

\section{Paul Saunders ${ }^{1}$}

${ }^{1}$ Canadian College of Naturopathic Medicine, Canada

Natural health products, primarily in the form of botanical medicines and homeopathic medicines were used in the treatment of over 20 cases of acute Covid-19. The botanicals included primarily Glycyrrhiza, Echinacea, and Andrographis. and the homeopathic medicines Bryonia, Gelsemium, Hepar, Pulsatilla, and Cocculus. Cases included pregnant females, children, adults and seniors, This presentation will describe a few of the clinical cases in more detail and provide a summary table of patients, symptoms, and treatments. Various vitamins, minerals, and nutritional support factors were use as immune support or as prevention in another group of clinical cases.

\section{Rationale for Quercetin as a Potential Supplement to Increase Resistance to COVID-19 Infection and to Reduce Symptoms During Recovery? \\ Julia Solnier ${ }^{1}$, Chuck Chang ${ }^{1}$, Mary Hardy², Roland Gahler ${ }^{3}$ \\ IISURA, Burnaby, BC, Canada \\ ${ }^{2}$ Academy of Integrative and Holistic Medicine, San Diego, California, USA \\ ${ }^{3}$ Factors Group of Nutritional Products Inc. R\&D, Burnaby, BC, Canada}

The potential of herbal medicines to affect outcomes of the novel coronavirus disease, COVID-19, has been much discussed by researchers throughout the pandemic. Quercetin represents one of the best studied flavonoids with numerous health-promoting effects such as antiviral, anti-inflammatory, anticancer, and cardiovascular protective 
activities. In particular, the anti-inflammatory capacity of flavonoids in viral diseases-such as that of quercetincan be of great value for the adjuvant therapy of COVID-19. In some studies, quercetin was found to reduce the release of inflammatory cytokines (e.g., IL-8) which may alleviate infection-related symptoms and suppress overwhelming inflammatory responses that are often associated with severe COVID-19 conditions. Furthermore, the antioxidative effects of quercetin preventing tissue damage by scavenging free radicals might be another benefit for the recovery process of viral diseases like COVID-19. Despite the fact that quercetin exerts a broad range of pharmacological activities including virucidal effects against many different viruses (e.g., herpes simplex, influenza and parainfluenza type 3), its poor pharmacokinetic profile strongly limits its clinical use. For this purpose, several galenic formulations have been introduced to improve the rate of absorption, as well as gastrointestinal solubility of quercetin supplementation. Development of a novel strategy, Quercetin Lipomicel, represents a superior delivery solution to manage insufficient bio-accessibility of quercetin and enhance the bioavailability of the compound. As the bioavailability of quercetin is one of the key limiting factors in its therapeutic use, this innovation could allow the compound to be more clinically effective. Novel carrier designs such as LipoMicel may present a powerful future strategy to circumvent low bioavailability issues of various poorly absorbable plant compounds. However, further research and more sophisticated clinical studies on the efficacy will be needed.

\section{Evaluation of Ethanol and Isopropyl Alcohol Content in Hand Sanitizers Samples Purchased from Retail Location in the Greater Vancouver Regional District \\ Palak Tank', Jamie Finley², Michael Chan², Paula N. Brown², Hong Sy² \\ ${ }^{1}$ British Columbia Institute of Technology, Biotechnology, Burnaby, British Columbia, Canada \\ ${ }^{2}$ British Columbia Institute of Technology, Centre for Applied Research \& Innovation, Burnaby, British Columbia, Canada}

According to the World Health Organization (WHO) alcohol-based hand sanitizer formulations are an effective alternative to handwashing methods to prevent the spread of coronavirus. Health Canada and the US Food and Drug Administration introduced interim measures that streamline processes for approval of hand sanitizers to help alleviate supply issues.

Minimum content requirements for ethanol and/or 2-propanol, medicinal ingredients in alcohol-based sanitizers, ensure the products are efficacious. In response to product warnings and recalls, the objective of this work was to validate a quality control test method and conduct a basket study of products in the local market. The validated Headspace Gas Chromatography- Mass Spectrometry (HS-GCMS) method measured ethanol and 2-propanol content in hand-sanitizer products and was used to screen for other possible contaminants. A total of 46 hand-sanitizer products were purchased from retail locations throughout greater Vancouver, B.C. and were analyzed and compared against Health Canada's ethanol content requirements. Of those samples tested, eight products did not meet Health Canada requirements. Two of the products were also found to contain ethyl acetate, an ingredient that could pose additional safety concerns. Products that have not been tested or fail to conform to Health Canada requirements could pose serious health risks and may not be effective at killing pathogens.

\section{Indigenous Traditional Medicine}

\section{A Meta-Analysis of Nishnaabeg Ethnobiology \\ Zonghua Ai ${ }^{1}$, William Johnson', Veronica King-Jamieson², Jonathan Ferrier ${ }^{3}$ \\ 'Department of Biology, Dalhousie University, Canada \\ ${ }^{2}$ Education and Awareness, Mississaugas of the Credit First Nation \\ ${ }^{3}$ Department of Biology, Dalhousie University; Board of Education, Mississaugas of the Credit First Nation, Canada}

Michi Saagiig, Nishnaabeg communities are revitalizing local food, medicine, and material cultural practices. Mississaugas of the Credit First Nation (MCFN) requested an ethnobiology study to assist post-Covid 19 community based ethnobiology. The majority of Nishnaabeg ethnobiology literature is outdated and requires updating via a standardized meta-analysis to provide accurate contributions to MCFN's educational programming. Our work aims to serve as a community reference for members learning and practicing traditional food, medicine, and material traditions while revitalizing Nishnaabemwin. The meta-analysis aims to support MCFN's specialist healers with critically endangered knowledge by providing cross-community consensus indices for insights into food and medicine safety and efficacy. We searched for studies published in any language from any date using library databases, vendors, 
and reference lists. Unique linguistics and Indigenous taxonomic nomenclature was maintained in the literature review data collection process. A meta-analysis of the standardized data was conducted for quantitative ethnobiological guidance and prioritizing future natural product research avenues. Consensus indices provided a metric for Nishnaabeg food and medicine safety and efficacy. To date 2633 use reports were organized into some previously unreported ethnobiological categories. This ongoing study provides the MCFN community with a contemporary baseline to assess and advance food, medicine, and material practices in a pragmatic, safe and effective way with contributions to MCFN's Nishnaabemwin dictionary.

\section{Traditional Indigenous Medicine and Health Systems Transformation in Abya Yala (Latin America) \\ Daniel Gallego-Perez ${ }^{1}$ \\ ${ }^{1}$ Boston University, USA}

Surviving healing traditions of native Indigenous peoples from the several civilizations across the Abya Yala, the territory that is most commonly referred to as 'Latin America' have been largely marginalized during colonial and post-colonial times. These healing arts have been in dynamic interplay with biomedical, African-derived, and other medical systems practiced across the region. The interplay, however, has been asymmetric, shaped by the colonial order and a myriad of social forces. Despite this asymmetry, Indigenous medical cultures in Latin America continued to co-exist, evolve, and thrive. Indigenous people's struggles for rights recognition and dignity at the national and international stages brought renewed attention to the importance of valuing and preserving their culture, traditions, and knowledge. Increased political recognition of Indigenous peoples brought an identity crisis in several Latin American countries, which created an opening to reshape the social contracts. These renewed frameworks of reference necessarily included indigenous cosmovision and principles, which permeated ongoing health reform efforts at the dawn of the twenty-first century. This presentation will outline the historical context that introduced traditional indigenous concepts into health systems transformation efforts in Latin America. Initiatives that the region's countries have developed to address the disgraceful health disparities they endure will be critically reviewed, emphasizing the attempts to articulate traditional indigenous medicine and biomedicine-oriented health systems.

\section{Gathering Medicine: A Skwxwú7mesh Perspective on the Role of Culturally Important Plants in Indigenous Resurgence \\ Leigh Joseph ${ }^{1}$ \\ ${ }^{1}$ Ethnobotanist, Squamish Nation, Canada}

In this talk, Styawat/Leigh Jo39seph, a Squamish Nation ethnobotanist will explore the links between plants, landbased knowledge and access and Indigenous resurgence and healing. Drawing on her experience working as an ethnobotanist in Indigenous communities in BC and the Yukon, Leigh will share perspectives and storytelling based on how culturally important plants play a central role in culture, health and wellbeing.

\section{The Protection of Indigenous Medical Knowledge by Legal Agreements Élisabeth Patterson ${ }^{1}$ \\ ${ }^{1}$ Dionne Shulze, Canada}

Indigenous medical knowledge is not well protected by law, whether in Canada or internationally. Western intellectual property mechanisms are not well adapted and new forms of protection are only being developed very slowly. In this context, protecting IMK through an agreement can be helpful.

We will briefly describe the available legal instruments, new developments and their challenges. We will then describe how knowledge holders and users can use an agreement to ensure the sharing of knowledge is done in a respectful and mutually beneficial manner. Such agreements generally include sections on: confidentiality of traditional knowledge, prior informed consent of knowledge holders and of appropriate authorities, measures to ensure $\mathrm{OCAP}^{\circledast}$ (ownership, consent, access and possession) principles are respected, joint authorship or recognition, shared ownership and benefits for the community. Close collaboration at all steps of the project is essential.

We will Highlight Lessons Learned, Based on Concrete Examples. 


\title{
The Investigation of Sooyaiaiihtsi, a Blackfoot (Nitsitapi) Medicinal Plant Extract and its Effects on Human Cancer Cells
}

\author{
Haley Shade1, Araba Sagoe-Wagner ${ }^{1}$, Donald Shade1, Roy M. Golsteyn ${ }^{1}$ \\ ${ }^{1}$ University of Lethbridge, Canada
}

The Natural Product Laboratory at the University of Lethbridge is located on the traditional homelands of the Blackfoot (Niitsitapi) people. Our laboratory has launched the Prairie to Pharmacy Program, in which some of prairie plants used by the Blackfoot people since time immemorial are investigated for their potential biological activity.

The Blackfoot medicinal plant material, Sooyaiaiihtsi, was extracted with either 75\% ethanolic/water (PP-1060A) or $100 \%$ dichloromethane (PP-1060B). By the MTT cell viability test using the colon cancer cell line HT-29, PP-1060A was moderately cytotoxic at an $\mathrm{IC}_{50}$ of $101 \mathrm{ug} / \mathrm{mL}$. PP-1060B was cytotoxic at an $\mathrm{IC}_{50}$ of $45 \mathrm{ug} / \mathrm{mL}$. The cell line U2OS (osteosarcoma) was less sensitive to either treatment with $\mathrm{IC}_{50} \mathrm{~s}>100 \mathrm{ug} / \mathrm{mL}$. We then applied PP-1060A or PP-1060B to U2OS cells at concentrations of $15,50,150$ or $500 \mathrm{mg} / \mathrm{mL}$ and observed them by phase-contrast microscopy at regular intervals. In parallel, cells were treated with the solvent alone, dimethylsulphoxide (NT). At $500 \mathrm{ug} / \mathrm{mL}$ treatment cells had died. Relative to NT cells, by 24 hours cells treated with PP-1060B at 50 or $150 \mathrm{mg} / \mathrm{mL}$ displayed distinct clear vesicles, suggesting a change in membrane trafficking. To determine the source of these vesicles, we treated U2OS cells with PP-1060B and incubated them with the fluorescent dye, Nile Red that accumulates in lipid droplets. Relative to control cells, the PP-1060B induced vesicles did not stain with Nile Red. We then treated cells with PP-1060B and Acridine orange, which accumulates in acidic vesicles. PP-1060B treated cells were enriched in Acridine orange staining vesicles, suggesting induction of lysosomal compartments or autophagy, a form of cell death. We are now investigating whether the PP-1060B extract prepared from Sooyaiaiihtsi induces autophagy in cancer cell lines. Our research reveals the potential for Blackfoot traditional medicines to be studied using the integration of both western science and traditional knowledge.

\section{Global Advances in NHP Research}

\section{Bio-based Additives for Food Application}

\section{Lillian Barros' ${ }^{1}$}

${ }^{1}$ Centro de Investigação de Montanha (CIMO), Instituto Politécnico de Bragança, Campus de Santa Apolónia, 5300-253 Bragança, Portugal

Natural sources have been increasingly explored for their countless beneficial effects on human health, especially by plants and mushrooms. Beyond their nutritional properties, which make them important components of a balanced diet, these matrices are also rich in bioactive molecules that exert important functions as antioxidant and antimicrobial agents. Given these properties, several researches have been focusing the development of bio-based ingredients, namely colouring, bioactive, and preservative additives, through sustainable practices and processes. From this point of view, the exploitation of food industry residues to produce such ingredients is an increasing concern, along with the use of green solvents in the extraction procedures, among others.

Several compounds extracted from plants and mushrooms have been applied in food matrices with different purposes. For example, betalains (e.g. gomphrenin II, gomphrenin III, isogomphrenin II, and isogomphrenin III) and anthocyanins (e.g. cyanidin, delphinidin, and malvidin derivatives) obtained from purple globe amaranth, rose, dahlia, centaurea, strawberry-tree, roselle, and blueberry have proved bioactive and colouring properties when incorporated in ice-cream, yogurt, and waffles. Moreover, phenolic acids (e.g. rosmarinic acid), flavonoids (e.g. quercetin derivatives), and ellagitannins (e.g. sanguiin H-10 and lambertianin) from mushrooms, wild strawberry, rosemary, mountain sandwort, and flowers of silva brava demonstrated bioactive properties when introduced in gelatin, yogurt, and cottage cheese. On the other hand, strawberry-tree, basil, lemon balm, sweet chestnut flowers, fennel, and German chamomile revealed to be great sources of preserving molecules with antioxidant and antimicrobial activity, such as flavonoids (e.g. catechin, and quercetin and luteolin derivatives), phenolic acids (e.g. rosmarinic, chicoric, lithospermic, caffeic, and caffeoylquinic acids), and hydrolysable tannins (e.g. trigalloyl-HHDP-glucoside), which were tested in loaf bread, cupcakes, yogurt, cheese, and cottage cheese.

The creation of new bio-based ingredients is, therefore, of great importance and justifies the focus of novel researches on improving their development in order to guarantee the sustainability of their application. 


\section{Interaction with Gut Microbiota is a Relevant Mechanism for Therapeutic Activity of Herbal Extracts}

\section{Rudolf Bauer ${ }^{1}$}

${ }^{1}$ Institute of Pharmaceutical Sciences, University of Graz, Austria

Gut bacteria are processing food, but also herbal medicinal products, and thereby can produce signalling molecules that regulate our body functions. Therefore, dysbiosis of gut microbiota can contribute to health problems, like systemic inflammation, asthma, obesity, diabetes, and even autoimmune diseases or certain forms of cancer. It is of special interest, which signalling molecules originate from herbal extracts, and how herbal extracts modulate our gut ecosystem [1-3].

We have established a research platform, in which predigestion of the upper intestinal tract is conducted in a static in-vitro model according to the InfoGest consensus method. Subsequently pre-digested extracts are incubated with human faecal suspension under anaerobic physiological conditions. Metabolization of the constituents of the herbal extracts are monitored by LC-HRMS. Microbiome shifts induced by the extracts are tracked by 16S RNA sequencing.

Predigestion regularly is not producing many new metabolites [4]. However, microbial degradation of many classes of compounds has been observed during incubation of various herbal extracts with human faecal suspension. Flavonoid glycosides and aglycones, procyanidins, salicylic alcohol derivatives, caffeic acid derivatives, and triterpene glycosides, are regularly rapidly and intensively metabolized. Numerous newly formed compounds could be assigned to putative microbial metabolites [5].

In conclusion, the combined next-generation sequencing and LC-HRMS approach allows straightforward detection of relevant microbiome shifts and metabolites. The role of the metabolites as active principles needs to be more investigated.

[1] Li H, Zhou M, Zhao A, Jia W. Phytother Res. 2009;23(9):1332-1335.

[2] David LA, Maurice CF, Carmody RN, Gootenberg DB, Button JE, Wolfe BE, Ling AV, Devlin AS, Varma Y, Fischbach MA, Biddinger SB, Dutton RJ, Turnbaugh PJ. Nature 2014;505(7484):559-563.

[3] Thumann TA, Pferschy-Wenzig EM, Moissl-Eichinger C, Bauer R. J Ethnopharmacol. 2019;245:112153.

[4] Thumann TA, Pferschy-Wenzig EM, Aziz-Kalbhenn H, Ammar RM, Rabini S, Moissl-Eichinger C, Bauer R. Phytomedicine. 2020; $71: 153221$.

[5] Pferschy-Wenzig EM, Koskinen K, Moissl-Eichinger C, Bauer R. Front Pharmacol. 2017;8:893.

\section{Metabolomics Tools for Authentication of Botanical Dietary Supplements}

\section{Nadja B. Cech ${ }^{1}$}

${ }^{1}$ Department of Chemistry and Biochemistry, University of North Carolina Greensboro, Greensboro (NC), USA

Botanical (plant-based) natural products, which fall under the classification of "natural health products" in Canada, and "dietary supplements" in the US, constitute a multi-billion-dollar industry in North America. Regulation and quality control for this industry is an ongoing challenge. Myriad examples exist whereby commercially available botanical natural products are either intentionally or unintentionally adulterated or mislabeled, a situation that constitutes a major health concern for consumers. Even for correctly identified botanical natural products, composition differs depending on myriad factors, including variability in genetics, cultivation conditions, and processing methods. While there is general agreement that rigorous scientific studies are needed to evaluate the safety and efficacy of botanical natural products used by consumers, researchers conducting such studies face the challenge of dealing with inherently complex mixtures of variable composition. Unfortunately, many studies of botanical natural products are carried out with poorly characterized study material, such that the results are irreproducible and difficult to interpret. To address these challenges, our research group has developed mass spectrometry metabolomics approaches to capture and compare the chemical diversity of complex botanical natural products. We have compared targeted and untargeted methods, with the goal of establishing a set of recommended approaches for effective selection of botanical material for research studies. This presentation will describe this process, an important element of which is the calculation of a "composite score", which is a quantitative metric that classifies the similarity among samples based on principal component analysis data. A case study will be presented illustrating how this approach has been applied to select study materials of kratom (Mitragyna speciosa) for clinical evaluation. 


\section{Cranberry as a Source of Novel Phytomelatonin Natural Health Products}

\section{Lauren A. E. Erland', Ryland T. Giebelhaus', Susan J. Murch'1}

${ }^{1}$ Department of Chemistry, University of British Columbia, Kelowna, BC, Canada V1V 1V7

Cranberry (Vaccinium macrocarpon) is the source of well established and effective natural products for the treatment of urinary tract infections, but the products can be highly variable and have not been well optimized. Our research demonstrated that freshly harvested cranberries contain high levels of melatonin. Melatonin supplements currently available in the market are derived from ovine or bovine sources or chemical synthesis and may contain up to 5 isomers, enantiomers and degradation products that are neurologically active chemical contaminants. Plant-sourced melatonin would provide a human, vegan and vegetarian friendly source of melatonin which may have a greater appeal to consumers. Additionally, the possibility for sourcing melatonin from traditionally considered waste products such as post-crush grapes or cranberries, would represent a novel additional income source for these industries in addition to a relatively low cost, safe, sustainable, and environmentally friendly source of melatonin. We used validated liquid chromatography-mass spectrometry methods to determine the environmental and harvest factors that affect melatonin contents in commercial cranberry and several related Vaccinium species. Our data show that melatonin is an important plant signaling molecule in Vaccinium species. Since melatonin is a plant signaling molecule, absolute concentrations are highly variable, conditions can be manipulated to increase melatonin levels in harvested tissues. Optimized growing, harvest and processing methods have the potential to produce cranberry fruit with up to $2.5 \mu \mathrm{g} / \mathrm{g}$ melatonin that serve as a suitable starting material for development of future phytomelatonin products.

\section{Pre-Clinical Pharmacology of Purified Plant Cannabinoids and Terpenes from Cannabis Sativa}

\section{Robert Laprairie', Kawthar A. Mohamed ${ }^{1}$, Ayat Zagzoog', Tallan Black ${ }^{1}$}

'University of Saskatchewan, Canada

The Cannabis sativa plant contains more than 120 cannabinoids. $\Delta^{9}$-tetrahydrocannabinol $\left(\Delta^{9}\right.$-THC) and cannabidiol (CBD) have been studied far-more extensively than the many other, less-abundant plant-derived (phyto) cannabinoids. The type 1 and type 2 cannabinoid receptors $(\mathrm{CB} 1 \mathrm{R}, \mathrm{CB} 2 \mathrm{R})$ are the best-characterized receptors of the cannabinoids. $\Delta^{9}$-THC is the psychoactive and intoxicating constituent of Cannabis through its partial agonism at CB1R, whereas CBD's pharmacology is far more complex. Our group has on ongoing interest in the fundamental pharmacology of phytocannabinoids and terpenes. To date, we have assessed the activity of 14 phytocannabinoids and 8 terpenes at human $\mathrm{CB} 1 \mathrm{R}$, and $\mathrm{CB} 2 \mathrm{R}$ in Chinese hamster ovary $(\mathrm{CHO})$ cells stably expressing these receptors and in $\mathrm{C} 57 \mathrm{Bl} / 6$ mice. Compounds are assessed for their affinity to receptors, ability to inhibit cAMP accumulation, Barrestin2 recruitment, receptor selectivity, and ligand bias in cell culture; and cataleptic, hypothermic, antinociceptive, hypolocomotive, and anxiolytic effects in mice. Our data reveal partial agonist activity for many phytocannabinoids and some terpenes tested at CB1R and/or CB2R, as well as in vivo responses often associated with activation of CB1R. When cannabinoids and terpenes are used in combination, many terpenes alter the efficacy of cannabinoids, supporting some aspects of an 'entourage effect'. Our data add to a rapidly growing field showing cannabinoid receptor-dependent pharmacology for phytocannabinoids and terpenes. Our hope is that these data better inform the safe medical and non-medical use of cannabis products, and lead to the development of novel therapeutics.

\section{Insights into the Pharmacological and Chemical Investigations of Three Popular Herbal Remedies ("Copalchi", "Insulina", and "Zacatechichi") for Treating Diabetes in Mexico}

\section{Rachel Mata ${ }^{1}$}

${ }^{1}$ Universidad Nacional autónoma de México

Herbal medicines are an essential element of alternative health care in Mexico, and the best testimony of their efficacy and cultural value is their persistence in contemporary Mexican markets where the highest percentage of medicinal and aromatic plants is sold. Considering the global and local situation of Type II diabetes mellitus, which has become one of the most significant health problems worldwide, the Mexican population uses more than 350 plants for treating diabetes. Among these herbal remedies, "copalchi" (Hintonia latiflora (Sessé \& Moc. ex DC.) 
Bullock, Rubiaceae), "insulina” (Salvia amarissima Ortega, Lamiaceae) and "zacatechichi” (Calea ternifolia Kunth, Asteraceae) are highly valued. The most important ethnobotanical, phytochemical and pharmacological studies on these species will be highlighted in this presentation. The quality control procedures developed for their unequivocal identification will be also summarized. These will be valuable to avoid partial adulteration or substitution for other species commonly designated with the same common names. Future studies are required to solve the puzzle around these species' toxicity and better understand their pharmacological actions.

\section{Discovering Anti-Cancer Natural Health Products and their Interaction with Standard Chemotherapeutic Drugs: Possibility of Using them as Adjuvant with Standard Treatment}

\section{Siyaram Pandey ${ }^{1}$}

${ }^{1}$ University of Windsor, Canada

Plant extracts and their active compounds have been used for medicinal purposes for centuries by various traditional medical practices including Ayurveda, Traditional Chinese Medicine. Interestingly, some of the most common anticancer drugs like taxol, vincristine are derived from plat extracts. Unfortunately treatment of metastatic advanced cancers using adjuvant therapies (multiple compounds) leads only to a limited success, but severe side effects. We have previously demonstrated very potent anticancer activity of different plat extracts including dandelion root, lemongrass, long pepper and white tea extracts. Since these extracts are safe and can be taken orally, the total extract (which is adjuvant of many compounds in itself) could be taken as supplements along with standard cancer treatments. One major hurdle is their potential interaction with standard chemotherapies and radiation therapy. Many oncologists strongly oppose taking any natural extracts as they think it could hamper the treatment of cancer patients. Therefore, it is critical to investigate drug- natural extracts interaction. Our hypothesis is that natural extracts would sensitize cancer cells to standard treatment as well as reduce toxicity related to these treatments for healthy tissue. We have demonstrated that Dandelion and Lemongrass extracts display positive interaction with different chemo regimens in colon and prostate cancer cell in vitro and in vivo. Furthermore, we have discovered that these extracts inhibit appearance of colon tumor in transgenic APC ${ }^{\mathrm{min}}$ mice indicating their preventative ability.

\section{Avocado-derived Avocadyne as an Inhibitor of Fatty Acid Oxidation to Selectively Eliminate Leukemia Cells}

\section{Matthew Tcheng ${ }^{1}$}

'University of Guelph, Canada

Acute myeloid leukemia (AML) is a devastating blood cancer characterized by the uncontrolled proliferation and accumulation of myeloid cells that fail to mature into functional blood cells. Severe treatment related co-morbidities triggered by chemotherapeutics that have remained virtually unchanged for the past four decades underscore the need for novel agents that are better tolerated by patients. Compared to normal blood cells, AML cells exhibit a distinct metabolic phenotype characterized by increased reliance on higher rates of fatty acid oxidation (FAO) for survival.

FAO inhibitors under investigation for anti-AML therapy either have not been tested in humans or are hepatotoxic, preventing clinical use. An unbiased respirometry-based screen identified, avocadyne (AYNE), a 17-carbon long trihydroxylated avocado-derived lipid with a terminal triple bond, as an inhibitor of the very long chain acylCoA dehydrogenase (VLCAD), an enzyme catalyzing long chain FAO. VLCAD was overexpressed and critical to leukemia mitochondrial metabolism.

Genetic knockdown or pharmacological inhibition of VLCAD activity hindered FAO, mitochondrial respiration, and FAO contribution to the TCA cycle resulting in decreased AML survival, proliferation, and long-term repopulation potential. ATP quantification as well as assessment of mitochondrial metabolism with stable isotope tracers demonstrated divergent cell fates in patient-derived AML and normal hematopoietic cells following VLCAD inhibition, indicating a potential therapeutic window for FAO targeting. In leukemia cells, FAO suppression at VLCAD did not trigger compensatory glycolytic metabolism, resulting in ATP depletion and AML cell death. In contrast, hematopoietic cells were spared with no interruption of mitochondrial metabolism and cell viability. AYNE was well-tolerated in mouse models and demonstrated in vivo efficacy by eliminating human AML cells while sparing normal blood cells. Together, these results elucidate the pre-clinical efficacy of FAO inhibition by avocadyne, exploiting a novel metabolic vulnerability in this devastating disease. 


\title{
NHPs in Clinical Practice and Research
}

\section{Opportunities to Utilize Natural Psychedelics as New Tools to Meet Mental Health and Wellness Needs}

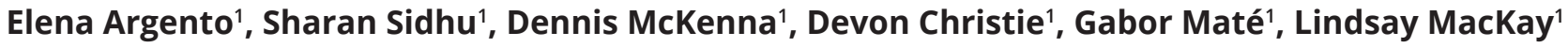 \\ ${ }^{1}$ Numinus Health \& Wellness, Canada
}

Mental health disorders are a leading cause of disability, affecting an estimated 1 billion people worldwide. In Canada, one in two people will have experienced a mental health condition by 40 years of age. Canadians also suffer among the highest rate of posttraumatic stress disorder (PTSD) worldwide, affecting close to $10 \%$ of the population. The limitations of current treatment options, alongside heightened post-pandemic demand for mental healthcare, underscores an urgent need for novel approaches to bolster existing therapies. Psychedelic plants and fungi, such as psilocybin-containing mushrooms and the Amazonian plant brew known as ayahuasca, have been used for centuries for a wide range of medicinal and healing purposes. Following studies throughout the 1950s-70s when psychedelics were considered to achieve major breakthroughs in psychotherapy, recent clinical trials have demonstrated the promising potential of psychedelic-assisted psychotherapy to enhance treatments for PTSD, depression, end-of-life anxiety, and substance use disorders. For instance, a randomized clinical trial (RCT) of psilocybin-assisted psychotherapy demonstrated effect sizes more than 4 times greater than pharmacological treatments for depression, while another double-blind RCT comparing psilocybin with escitalopram, a selective serotonin reuptake inhibitor (SSRI), found several outcomes favoring psilocybin. Similarly, a Brazilian RCT demonstrated significant and rapid antidepressant effects following a single dose of ayahuasca compared to placebo. An emerging opportunity lies in advancing clinical investigation of natural psychedelics, such as whole mushroom extracts, which may have several advantages over their synthetic counterparts. Consistent historical and clinical findings, which have demonstrated overall safety and efficacy of psychedelics, encourage further research on natural psychedelics to explore broader chemical constituents and formulations. This presentation aims to provide an overview of the research on naturally occurring psychedelic substances and opportunities to utilize psychedelics to address highly prevalent mental health disorders and bring about lasting benefits to health and wellbeing.

\section{Industry-Sponsored Clinical Trials to Support Regulatory Requirements}

\section{Andrew Charrette ${ }^{1}$}

${ }^{1}$ KGK Science, Canada

Health products from NHPs to biologic drugs require pre-market regulatory review to demonstrate their safety and substantiate efficacy. Designing and executing a clinical trial that meets the regulatory requirements and business objectives set out by the sponsoring firm can be complex. Given the significant capital investment required to conduct clinical research, there is limited room for error. This is especially true for cannabis health products and psychedelic therapeutics as they expand the horizons of naturally-sourced health ingredients into uncharted regulatory waters. This presentation delves into some of the important considerations that industry sponsors should consider when embarking upon conducting clinical research to support safety and efficacy claims for these consumer products. With a focus on regulatory filings and claims substantiation, real-world examples are used to examine the factors that go into designing successful clinical trials for naturally sourced health products and how to avoid common pitfalls.

\section{Cannabinoid-Based Medicines in Canada: Clinical and Product Data at A Leading Medical Cannabis Clinic}

\author{
Erin Prosk ${ }^{1}$, Lucile Rapin ${ }^{1}$, Maria-Fernanda Arboleda' ${ }^{1}$, Michael Dworkind ${ }^{1,2}$ \\ 'Santé Cannabis, Montreal, QC, Canada \\ ${ }^{2}$ Department of Family Medicine, McGill University, Canada
}

Medical cannabis, or cannabinoid-based medicines (CBMs) have gained global popularity driving evolution of regulatory access frameworks in more than 30 countries to date. CBD products have gained significant attention with claims of efficacy for chronic pain, anxiety, depression, poor sleep and overall quality of life. While evidence is limited, experienced clinicians often initiate patients on oral CBD products to reduce risk of adverse effects associated 
with THC. Controlled data collection at cannabis clinics provides an opportunity for Real-World Evidence, especially for difficult to treat conditions such as refractory pain.

Data analysis assessed adult patients who initiated treatment with CBMs for the treatment of pain at a network of four medical cannabis clinics in Québec, Canada between July and November 2020. Clinical and product data is collected via an internal protocol with continued improvements to product details, safety data and effectiveness using validated tools. Patients experienced pain refractory to traditional medications with additional secondary symptoms. Effectiveness between baseline and FUP was assessed using paired t-tests with a p-value set at $\mathrm{p}=0.05$.

At initial visit, a large majority of patients were treated with plant-derived cannabis products. Oral cannabis oil was preferred with Cannabidiol (CBD)-rich products predominantly prescribed (55.6\%) followed by THC:CBD balanced (41.4\%). Average daily dosage of oral CBD was $12.6 \mathrm{mg}$ (range 2-63 mg) and THC $2.3 \mathrm{mg}$ (range $0.07-18 \mathrm{mg}$ ). There was statistically significant (all ps $<0.04$ ) improvement between Baseline and FUP for all pain variables and for all symptom variables except nausea.

These results provide preliminary indication that CBMs may be considered as an adjunct treatment for refractory pain. In contrast with current literature, oral CBD-rich products were the preferred product and method of administration, requiring additional study to support future regulatory changes.

\section{Exploring Clinical Trials Involving NHPs Dugald Seely ${ }^{1}$ \\ ${ }^{1}$ Centre for Health Innovation, Canada}

Clinical trials can be challenging to develop and execute and particularly so when it comes to the application of multiple natural health products. In this talk, aspects of clinical trial development will be reviewed including preparation, gathering a team of investigators, population and sample size determination, selecting the intervention and resolving which outcomes are best applied. Beyond development, the conduct and execution of a series of clinical trials will be discussed that involve natural health products for specific conditions. Points relating to enrolment, application of the intervention, adherence, and follow up are considered through the lens of three distinct trials. These examples including cancer patients and people with a proven positive COVID-19 infection, portray different designs from the simplest placebo controlled monotherapeutic controlled trial to a multi-therapeutic multi-modality pragmatic RCT. The examples are chosen to explore the complexities and opportunities afforded through clinical research with projected vs actual realities discussed as they relate to timeline, recruitment and conduct.

\section{The Ayurvedic NHPs: Promise for Future Asmita Wele ${ }^{1}$}

${ }^{1}$ Bharati Vidyapeeth Deemed to be University, Pune, India

The relationship of humans and foods is eternal so as the knowledge of its variety and tastes. The therapeutic effects of foods have been explored in Ayurveda, the traditional Medicine of Indian subcontinent. Its health pyramid starts with foods, medicated foods, and medicines. The principles of taste, amount, proportion, processing, compatibleincompatible foods, rationale-irrational diet combinations, nutritional value, effects and adverse effects after long term use of specific foods are well studied in the Ayurvedic domain. The epigenetic factors associated with regional diet and habitual combinations; individual choices according to one's constitution (prakriti); satiety; diet to suffice specific physiological conditions like pr egnancy, adolescence and geriatrics; nutritional needs for particular purposes like sports, bodybuilding and spirituality are also identified. The concept of well-balanced diet and the foods that support healing during variety of diseases is explicitly studied. The outcome for the hypothesis that 'origin of disease lies in long term unhealthy food habits due to impairment of metabolic process (agni) has been incorporated with every major disease pathophysiology. Further, disease specific dietary guidelines, use of functional foods, concept of food-drug interaction and spices as nutraceuticals are also studied. The major issues regarding functional or therapeutic foods and nutraceuticals from Ayurveda about the quality, stability, inherent contradiction with fresh versus preserved, and measurement of personalised responses, which need to be addressed. Disease models to analyse role of food in rate of recovery can be a promising area of research. With the ever changing technology and deepening of our understanding regarding interlinking of immunity and food, post the pandemic, it is more than necessary to look at medicated foods with different perspectives. Also the compatibility of variety of NHPs from different geological zones on diverse ethnic populations should be studied using molecular methods and population studies. 


\section{Regulation, Policy, Commerce}

\section{The Role of Targeted Funding Authority in Natural Health Product Research Joseph Betz'}

${ }^{1}$ Acting Director of the NIH Office of Dietary Supplements, USA

The Dietary Supplement Health and Education Act (DSHEA) amended the U.S. Food, Drug, and Cosmetic Act and also established the Office of Dietary Supplements (ODS) within the NIH. Passage of the DSHEA assured consumer access to supplements, defined dietary supplements as food, laid out a regulatory framework enforced by the U.S. FDA, and provided a basis for supporting and disseminating research to strengthen knowledge of dietary supplements.

As directed by Congress, ODS's mission to foster an enhanced quality of life and health is achieved by providing resources for evaluating scientific information, stimulating and supporting research, disseminating research results, and educating the public. The ODS budget has grown from less than US \$1 million in 1995 to about \$25 million in 2020. This amount is modest by NIH standards, but ODS effectively leverages its resources with other parts of the $\mathrm{NIH}$ and other government agencies to create a disproportionately large impact dietary supplement research.

Over the past 25 years, ODS and its partners have created the NIH Centers for Advancing Research on Botanical and other Natural products program (CARBON), an Analytical Methods and Reference Materials Program, an Evidence-based Review Program, a Population Studies Program, and a new Resilience Program. The Office has also created public databases of dietary supplement labels, ingredients, and research, and supplement fact sheets. Other initiatives include creation and oversight of an international program for standardization of vitamin D metabolite measurements, support of measurement of total nutrient intake through the National Health and Nutrition Examination Survey and similar activities, and a program for studying nutrient biomarkers. Finally, the Office of Dietary Supplements has an active grant co-fund program. Going forward, ODS will continue to pursue its Congressionally mandated mission by continuing successful programs and by creating new programs as emerging needs are identified.

\section{Continuous Improvements to Regulatory Oversight of Natural Health Products in Canada}

\section{Natalie Page 1}

${ }^{1}$ Director General, Natural and Non-Prescription Health Products, Health Canada

Canada has one of the best regulatory systems in the world for natural health products, with some of the most stringent requirements. Health Canada's Natural and Non-prescription Health Products Directorate (NNHPD) authorizes natural health products for sale in Canada, supporting Canadians' access to a wide range of products for which safety, efficacy and quality standards are in place. Health Canada is committed to continuous improvement of its regulatory program to protect the health and safety of Canadians. In doing so, Health Canada ensures an active role in the research community through continued collaboration with Natural Health Product Research Society (NHPRS).

Acknowledging the importance of a strong collaboration between the regulator and the research community, this presentation will address key regulatory and policy initiatives currently underway, as well as Health Canada's forward agenda. Discussion will include areas where improvements are needed, informed by findings of the recent audit by the Commissioner of Environment and Sustainable Development.

A shared understanding of the key drivers for change and the forward policy agenda will enable increased opportunities for continued collaboration between Health Canada and NHPRS, including opportunities to leverage the expertise of NHPRS and its members to inform efforts to strengthen the Natural Health Products Program.

Améliorations continues de la surveillance réglementaire des produits de santé naturels au Canada

Le Canada possède l'un des meilleurs systèmes de réglementation au monde pour les produits de santé naturels, avec certaines des exigences les plus strictes. La Direction des produits de santé naturels et sans ordonnance (DPSNSO) de Santé Canada autorise la vente de produits de santé naturels au Canada, favorisant ainsi

l'accès des Canadiens à une vaste gamme de produits pour lesquels des normes d'innocuité, d'efficacité et de qualité sont en place. Santé Canada s'est engagé à améliorer continuellement son programme de réglementation afin de protéger la santé et la sécurité des Canadiens. Ce faisant, Santé Canada s'assure de jouer un rôle actif dans le milieu 
de la recherche par une collaboration continue avec la Société canadienne de recherche sur les produits de santé naturels (SCRPSN).

Reconnaissant l'importance d'une solide collaboration entre l'organisme de réglementation et le milieu de la recherche, cette présentation traitera des principales initiatives réglementaires et politiques en cours, ainsi que du programme d'avenir de Santé Canada. La discussion portera notamment sur les domaines où des améliorations sont nécessaires, à la lumière des conclusions de la récente vérification effectuée par le commissaire à l'environnement et au développement durable.

Une compréhension commune des principaux facteurs de changement et du programme politique à venir permettra d'accroître les possibilités de collaboration continue entre Santé Canada et la SCRPSN, y compris les possibilités de tirer parti de l'expertise de la SCRPSN et de ses membres pour éclairer les efforts visant à renforcer le Programme des produits de santé naturels.

\section{Contributed Sessions}

\section{Cancer}

\section{Identification of Distinct Types of Mitotic Arrests Induced by Prairie Asteraceae Plant Extracts in Human Cancer Cells}

\section{Haley Allard', Roy M. Golsteyn ${ }^{1}$}

${ }^{1}$ Natural Product and Cancer Cell Laboratories, University of Lethbridge, Canada

In the Prairie to Pharmacy Program we investigate endemic prairie plant species for natural products. The Asteraceae plant family is a large and widely distributed plant family in Canada and is related to plants that are the source of several natural product medicines. Previous work in our laboratory has shown that prairie Asteraceae species, Gaillardia spp. and Hymenoxys spp. contain sesquiterpene lactones that induce a cell cycle arrest during mitosis. During investigation of extracts prepared from different species, we identified several with anti-mitotic activity but observed subtle differences in how the cells arrested. We explored these differences to provide insight into natural products, mitotic arrest, and future projects for chemical isolation. We treated HT29 cancer cells with extracts from Arnica cordifolia, Arnica fulgens, and Ratibida columnifera, and compared their activity to a Gaillardia aristata extract that contains a sesquiterpene lactone called pulchelloid A. We used immunofluorescence microscopy to investigate the mitotic spindle after treatment with anti- $\alpha$-tubulin and anti- $\gamma$-tubulin antibodies, which are essential proteins for cell division, and anti-phospho-histone $\mathrm{H} 3$ antibodies to identify mitotic arrest. Cells treated with A. cordifolia, A. fulgens, and R. columnifera extracts arrested HT29 cells in mitosis, and displayed distorted microtubules. These distortions were different than the distortion caused by the G. aristata extract. We also observed three centrosomes in A. fulgens-treated mitotic cells, and loosely organized centrosomes in cells arrested in mitosis by G. aristata. These observations are distinct from the centrosomes observed in normal mitotic cells. These visual observations can be graphically displayed by analyzing the pixel density of photographed cells and reveal the differences in mitotic arrests. The differences in mitotic arrests suggest that the chemicals are inhibiting different protein targets. Chemicals such as these from prairie Asteraceae species have potential to be used as scientific tools or tested further for medicines in cancer treatments.

\section{A Novel Mango Ginger (Curcuma amada) Bioactive, 2,4,6-trihydroxy-3,5- diprenyldihydrochalcone, Inhibits Mitochondrial Metabolism to Impart Anti-Cancer Activity in Combination with Avocatin B

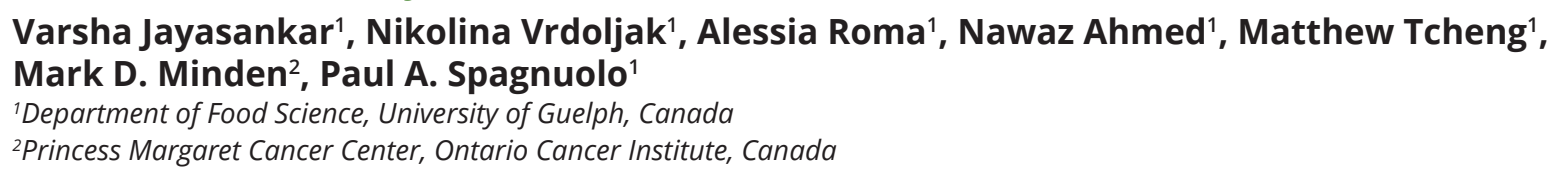

Acute myeloid leukemia (AML) is an aggressive malignancy of the blood and bone marrow in dire need of novel therapies. Nutraceutical compound Avocatin B (Avo B) is an avocado-derived polyhydroxylated fatty alcohol that has been identified as an inhibitor of fatty acid oxidation (FAO) in AML. The roots of the mango ginger plant, 
Cucuma amada, also contain a bioactive molecule identified as 2,4,6-trihydroxy-3,5-diprenyldihydrochalcone (M1), which possesses anti-microbial activity. In this study, M1 and Avo B combinations were tested for potential effects on AML in vitro.

M1 $(0.16-0.63 \mu \mathrm{M})$ and Avo B $(2.0 \mu \mathrm{M})$ were observed to synergistically reduce leukemia cell growth. Combination index (CI) values, as determined using Calcusyn software, were $0.6,0.65$, and 0.8 , indicating statistical synergy (i.e., $\mathrm{CI}<1)$. In addition, antagonistic combinations $(\mathrm{CI}>1$ ) were noted when $\mathrm{M} 1$ was combined with doxorubicin and cytarabine. In cells resistant to FAO, the combination was ineffective at reducing leukemia cell viability, however M1 remained active. Mechanistically, M1 was shown to inhibit complex I of the mitochondrial complex chain, suggesting that both molecules target altered leukemia cell metabolism. Taken together, M1 and Avo B is a novel combination with activity in AML worthy of further exploration.

\section{Sulforaphane Exhibits Anti-cancerous Effects on U251,U87 Glioblastoma Cells in Culture \\ Mostafa Khairy ${ }^{1}$ \\ 'University of Alberta, Canada}

Sulforaphane is a natural compound that occurs in broccoli sprouts. Several reports describe that sulforaphane induces apoptosis specifically in cancer cells but not in normal cells at the same dose. In vivo, sulforaphane is metabolized to sulforaphane $\mathrm{N}$-acetyl cysteine which has the ability to cross the blood brain barrier. Our research aims to test sulforaphane anti-cancer activity in glioblastoma cell lines, determine the dosage boundaries for toxicity to glioblastoma cells compared to normal cells, and identify the mechanisms that lead to the induction of cell death in glioblastoma cell lines. We demonstrated that sulforaphane had a cytotoxicity effect with an LD50 of $75 \mu \mathrm{M}, 40 \mu \mathrm{M}$ for U251, U87 respectively. A dose-response effect was observed. Sulforaphane at these concentrations also exhibited significant interference with cell invasion. Sulforaphane is therefore an excellent candidate for further exploration in this uniformly fatal brain cancer.

\section{Rasoushadhies: Herbo-Mineral Preparations-A Shaft of Light in the Field of Cancer Pavan Kulkarni ${ }^{1}$ \\ ${ }^{1}$ Bharati Vidyapeeth, Pune, India}

Rasoushadhies are herbo-mineral formulations that despite toxicity concerns in many countries are traditional treatments of choice by Ayurvedic Physicians in South Asia due to their tastelessness, smaller dosage, fast action and broader indications. Many compound formulations contain components like diamond, tourmaline, mica, arsenic, copper, gold, silver and sulphur which are used in cancer and may act as anti-cancer agents. Anti-cancer activity of these rasoushadhies may be associated with their cytotoxic action, apoptopic activity, cell mediated immunity, enzyme activation, cell protective activity, etc. In this presentation, rasoushadhies used in conditions of cancer will be described highlighting their probable mode of action in terminating the process of cancer pathogenesis.

\section{White Tea and Rosemary Extracts Selectively Induce Cell Death and Enhance the Efficacy of Common Chemotherapeutics in Triple-Negative Breast Cancer Chris Raad', Lauren Miles' ${ }^{1}$ Gabrielle Walach'1, Siyaram Pandey ${ }^{1}$ \\ 'University of Windsor, Canada}

While chemotherapeutics are effecitve, they are not selective in their mechanism of action, leading to adverse effects. Given the systemic toxicity associated with these therapies, they are not well suited for long-term use. Natural Health Products, or NHPs, may provide a way to selectively target the oxidative and metabolic vulnerabilities in cancer cells. White Tea (Camelia sinensis) and Rosemary (Salvia rosmarinus) are two natural extracts that have been studied extensively for their medicinal properties. However, their anti-cancer activity and mechanism of action are yet to be fully elucidated. We have examined the extracts' cancer-cell killing ability as well as their interactions with common chemotherapeutics in MDA-MB-231 cells, a triple-negative breast cancer cell line. We have demonstrated that White Tea Extract (WTE), an enhanced composition of White Tea, and Rosemary Extract (RE) selectively induce apoptosis in breast cancer cells with dose and time dependency. More importantly, the administration of the extracts in 
conjunction with cisplatin and paclitaxel sensitizes cancer cells to achieve enhanced cell death. Our findings suggest that WTE induces apoptosis by targeting cancerous mitochondria. In addition, RE does so by inducing oxidative stress in cancer cells. In sum, WTE and RE are well-tolerated treatments with powerful anti-cancer activity, thereby making them exciting candidates for providing long-term care to breast cancer patients.

\title{
Shikonin Inhibits Mitochondria Complex II to Selectively Eradicate Acute Myeloid Leukemia Cells
}

\author{
Alessia Roma', Matthew Tcheng' ${ }^{1}$ Nawaz Ahmed', Sarah Walker', Preethi Jayanth', \\ Mark D. Minden², Kristin Hope², Praveen P. Nekkar Rao ${ }^{3}$, Jessica Luc ${ }^{3}$, Andrew C. Doxey ${ }^{3}$, \\ Julie A. Reisz ${ }^{4}$, Angelo D'Alessandro ${ }^{4}$, Paul A. Spagnuolo ${ }^{1}$ \\ 'Department of Food Science, University of Guelph, 50 Stone Rd. Guelph Ontario, Canada N1G 2W1 \\ ${ }^{2}$ Princess Margaret Cancer Center, Ontario Cancer Institute, 610 University Ave, Toronto, Ontario M5G 2M9, Canada \\ ${ }^{3}$ University of Waterloo, 200 University Ave. W. Waterloo, Ontario, Canada N2L $3 G 1$ \\ ${ }^{4}$ Department of Biochemistry and Molecular Genetics, University of Colorado School of Medicine, Aurora, CO, USA
}

Acute myeloid leukemia (AML) is a disease of the blood and bone marrow with limited therapies. AML cells exhibit several metabolic alterations, including an increased reliance on oxidative phosphorylation and the respiratory electron transport chain (ETC). Activity of ETC complex II (CII) is elevated in AML and various therapies which indirectly target CII preferentially eradicate AML cells. Despite this, direct CII inhibition has not been previously studied in AML. Moreover, currently available CII inhibitors have high EC50s and reported toxicity. As such, we sought to identify novel CII inhibitors through an in silico screen and computational modelling. Through this study, we identified shikonin, a 1,4-naphthoquinone derived from the roots of Lithospermum erythrorhizon as a CII inhibitor. Shikonin reduced growth in AML cell lines (EC50: $1.0 \mu \mathrm{M} \pm$ 0.04) and patient-derived cells while sparing normal hematopoietic cells. In an in vivo model of AML, shikonin (3 mg/kg b.w.) significantly reduced (>50\%) the engraftment of primary cells and was well-tolerated. Mechanistically, shikonin-induced CII inhibition impaired mitochondrial respiration and tricarboxylic acid (TCA) cycle function, preferentially depleting ATP and aspartate in AML cells. In contrast, normal hematopoietic cells evade death by augmenting glycolytic capacity and reprogramming the TCA cycle, to maintain ATP and aspartate levels upon shikonin treatment. Taken together, these results highlight the specificity of CII targeting and demonstrate the potential utility of shikonin as a novel anti-AML agent.

\section{Evaluation of Anti-Cancer Activity, Elucidation of Drug-Drug Interactions and Mechanism of Action of Rosemary Extract in Colorectal Cancer

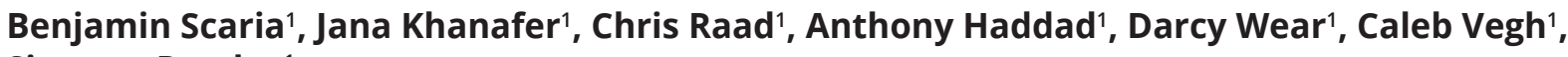 Siyaram Pandey ${ }^{1}$ \\ ${ }^{1}$ University of Windsor, Canada}

Colorectal cancer (CRC) is one of the most prevalent forms of cancer, accounting for 26,900 cases and 9,700 deaths in Canada, in 2020. Although conventional therapy contributes to the $65 \% 5$-year survival rate, toxic side effects of non-selective treatments, such as chemotherapy, remains a major issue. Natural health products (NHP's) are known to have anticancer properties, such as anti-proliferative and anti-inflammatory effects, and have been shown to induce anti-cancer effects in various cancer models. Specifically, rosemary extract (RE) contains polyphenolic compounds, such as rosmarinic acid, that have anti-cancer properties. Although RE has been shown to have selective anti-cancer activity, drug-drug interactions with chemotherapeutics have not been elucidated, but remain a major point of concern for oncologists. In this study, we evaluate the anti-cancer activity of a novel RE preparation by our industry partners, Synthite Industries. The primary objectives were to evaluate the nature of drug-drug interactions between RE and FOLFOX (commonly used chemotherapeutic in CRC), elucidate the mechanism of action of anti-cancer activity, and conduct an in-vivo study. The results indicate that RE is able to decrease cell viability and induce apoptosis in a dose-dependent manner. However, no significant effect on cell viability was observed in the normal colon mucosal cell line when treated with RE, suggesting that apoptotic induction is selective. No negative interactions were observed when cancerous cells were treated in combination with RE and FOLFOX, and some positive effects were observed. Furthermore, RE destabilized mitochondria by depolarizing their membrane potential selectively in cancerous cells, suggesting a possible mechanism for apoptotic induction. Currently, we are conducting an in-vivo study using a xenografted mouse model to further assess the efficacy of RE. 


\section{Characterizing the Anti-Cancer Efficacy of Paradise Tree Extract (PTE) Alone and in Combination with Standard Chemotherapeutics Against Human Melanoma}

\section{Siddhartha Sood', Mansi Arora', Karthik Baskaran'1, Siyaram Pandey ${ }^{1}$}

${ }^{1}$ Department of Chemistry \& Biochemistry, University of Windsor, Canada

Skin cancers such as melanoma are the most common form of cancer in the world. When treating patients, surgical resection and chemotherapy are two options traditionally used. However, surgery has shown success only in early-stage melanoma and current chemotherapeutics have limited efficacy and high non-specific toxicity. Thus, there is a need to discover novel complementary therapies for use alongside standard chemotherapeutics. Natural extracts have been scientifically validated for anti-cancer efficacy in the past. Paradise Tree Extract (PTE) from the Simarouba glauca plant, known as "Lakshmi Taru" traditionally in India, is one such extract that has been shown to possess anti-cancer activity. In previous studies, it has been evaluated to show anti-cancer potential on colorectal cancer and leukemic cancer cell lines. However, it has not been investigated for use against human melanoma. Thus, for the first time, we characterized PTE efficacy in human melanoma cell lines A375 and G361 and its selective induction of apoptosis. In addition, the cellular mechanisms behind its anti-cancer activity were elucidated. Furthermore, the interaction of PTE with standard chemotherapeutics was evaluated, specifically involving taxol and MTIC prodrugs such as temozolomide and dacarbazine (currently the only approved single-agent chemotherapy for melanoma). The results indicate there is no negative interaction with these drugs and in the case of MTIC prodrugs, the potential for synergism. Overall, the results of this study indicate that PTE has the potential to be used as a selective and efficacious melanoma treatment either alone or in combination with current standards of care.

\section{Impact of an Avocatin-B Containing Diet on Leukemia Relapse}

\section{Sarah Walker ${ }^{1}$}

'University of Guelph, Canada

Acute myeloid leukemia (AML) is a devastating cancer with poor patient outcomes and suboptimal chemotherapy that results in high rates of relapse. Novel therapeutic interventions are needed.

Avocatin B (AvoB) is an avocado-derived bioactive compound which has been shown to selectively target leukemia cells by inhibiting fatty acid metabolism. Previous in vivo engraftment mouse models showed a reduction in human leukemia cell engraftment after injection of an AvoB emulsion; AvoB was also shown to synergize with cytarabine. Since AvoB can be found in the diet, this study evaluated whether dietary AvoB can impart anti-AML activity when provided as a post induction therapy.

Avocado powder containing AvoB was incorporated into mouse chow, and the kinetics of AML growth in the bone marrow was tracked through multiple bone marrow aspirates. After engraftment was established, mice were treated with $(50 \mathrm{mg} / \mathrm{kg})$ cytarabine and placed on control or AvoB containing diets. At study termination (64 days), mice on AvoB containing diets had a significant reduction in bone marrow repopulation compared to mice on control diets $\left(\mathrm{t}_{16}=2.3 ; \mathrm{p}<0.05\right)$. Moreover, the exponential growth rate was significantly delayed in mice on AvoB diets (CTL: $0.053 \pm 0.016 v s$. AvoB: $0.022 \pm 0.01 \mathrm{~min}^{-1}$ ). Together, this suggests that AvoB containing diets may be useful to delay leukemia relapse post-induction chemotherapy.

\section{Cancer Selective Induction of Cell Death by Piper Iongum and Camellia sinensis Extracts in Neuroblastoma and Glioblastoma: Mechanism of Action and NHP-Drug Interactions}

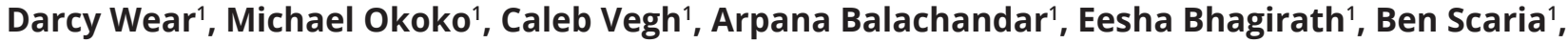 Siyaram Pandey ${ }^{1}$}

${ }^{1}$ University of Windsor, Canada

American statistics estimate that approximately 25,000 new nervous system cancer cases will be diagnosed in the USA in 2021, with $\sim 19,000$ deaths. Neuroblastoma is the most common tumour in infants whereas glioblastoma is generally diagnosed in adults with a very poor prognosis and survival rate. Current treatments for these cancers include surgery, which can often be difficult in the brain or in young children, as well as radiation and chemotherapy which are nonspecific treatments resulting in high toxicity to healthy cells and severe long-term side-effects. These tumours often develop chemoresistance and aggressive relapse occurs resulting in death in most cases. Alternatively, natural extracts 
known to be safe for consumption could be utilized to selectively target cancer cell growth. Specifically, extracts of Piper longum or Long Pepper Extract (LPE) and Camellia sinensis (Synthite Tea Extract or STE) have previously demonstrated selective anti-cancer properties with little to no effect on healthy cells. Indeed, we evaluated their anti-cancer efficacy and observed high levels of apoptotic induction in both human glioblastoma and neuroblastoma cell lines treated with LPE or STE. Interestingly, glioblastoma cells which demonstrate extreme resistance to the current chemotherapy temozolomide underwent significant apoptosis following LPE and STE treatment. More importantly, STE treatment in combination with temozolomide or cisplatin demonstrated strong positive interactions leading to enhanced cancer cell death with no affect on non-cancerous cells. Thus, STE has the potential to be used safely as a supplement to the standard chemotherapy to improve clinical outcomes. Mechanistically, LPE and STE target different vulnerabilities of cancer cells; LPE induces oxidative stress while STE destabilizes mitochondria. These results show potential for a combination treatment with enhanced anti-cancer activity of these NHPs and chemotherapies. Further work involving in-vivo studies could lead to the development a safe and selective treatment against neuroblastoma and glioblastoma.

\title{
Cannabis
}

\section{What's in a Name? Delving into the Cannabinoid Contents of Varying Cannabis Strains}

\author{
Anna Boukina1, Ryan Pusiak¹, Adam Waldbillig', Manu P. Gangola², Rubens L. G. Coelho², \\ Cory S. Harris ${ }^{1}$ \\ 1 University of Ottawa, Canada \\ ${ }^{2}$ The Flowr Group (Okanagan) Inc., Canada
}

As various parts of the world move forward with cannabis legalization, a fundamental issue remains debated by the scientific community: the classification of this chemically complex substance. Many important biologically active compounds are not accounted for in the current classification system, based largely on poorly characterized or standardized strains. This system focuses on morphological properties and uses popular terminology "sativa" and "indica". There is an urgent need to reevaluate the classification of this plant to ensure chemical consistency for medical-grade products to patients and recreational users. A proposed alternative approach to classification uses chemically distinct groups of cannabis variants named chemovars. This study aims to use the chemovar approach to investigate product similarities and differences between cannabis varieties, also known as strains. Commercially available, drug-type, Cannabis sativa strains were extracted, analyzed by HPLC with diode array detection, then examined using multivariate statistical analysis methods to determine if samples with the same strain name were chemically consistent with each other. Seven cannabinoid levels (acid + neutral forms) from 35 strains were measured and used for analysis: THC, CBD, THCV, CBDV, CBG, CBN, CBC. Using Principal Component Analysis, one-way Analysis of Variance (ANOVA), and Multi-Way ANOVA, two clusters of chemo-taxonomically indistinguishable strains were identified. Many other compounds exist within cannabis, such as terpenoids, that could improve groupings. This study demonstrates that chemical similarity exists between different cannabis strains suggesting that the current classification system does not properly represent the chemical variation when looking at seven cannabinoids. Although this study provides evidence that the chemovar approach is an improvement to the cultivar approach of classification there remain gaps that need to be addressed in a newly defined system.

\section{Secondary Metabolites Profiled in Cannabis Inflorescences, Leaves, Stem Bark, and Roots for the Development of Novel Natural Product}

Dan Jin ${ }^{1,2}$, Jacqueline Shan'2, Jie Chen ${ }^{1,3}$

${ }^{1}$ Department of Biomedical Engineering, University of Alberta, Edmonton, Alberta, Canada

${ }^{2}$ PBG BioPharma, Leduc, Alberta, Canada

${ }^{3}$ Department of Electrical and Computer Engineering, University of Alberta, Edmonton, Alberta, Canada

The legal cannabis market is growing quickly in North America. Currently in most cannabis growing facilities, only the flower tops are harvested because they contain the highest amount of the psychoactive tetrahydrocannabinol (THC) and non-psychoactive cannabidiol (CBD). Most other plant parts are discarded. This is a potentially unnecessary waste. Cannabis leaves, stem bark, and roots have the potential to be developed into novel natural health products and even medications. The aim of this study is to leverage a comprehensive investigation 
of chemical profiles in each plant part. The metabolites of the study included 14 cannabinoids, 45 terpenoids, 7 flavonoids, 3 sterols and 3 triterpenoids.

This multipart study included the development of quantitative methods using LC-MS for cannabinoids, LC-UV-MS for flavonoids, and GC-MS for terpenoids and sterols.

Cannabis inflorescence was characterized by cannabinoids (15.77-20.37\%), terpenoids (1.28-2.14\%), and flavonoids $(0.07-0.14 \%)$; the leaf by cannabinoids (1.10-2.10\%), terpenoids $(0.13-0.28 \%)$, and flavonoids $(0.34-0.44 \%)$; stem bark by sterols $(0.07-0.08 \%)$ and triterpenoids $(0.05-0.15 \%)$; roots by sterols $(0.06-0.09 \%)$ and triterpenoids $(0.13-0.24 \%)$.

This comprehensive profile of bioactive compounds can form a baseline of reference values useful for research and clinical studies to understand the "entourage effect" of cannabis as a whole, and also to rediscover therapeutic potential for each part of cannabis from their traditional use by applying modern scientific methodologies.

\section{Compositional Analysis of Cannabis Extracts to Support Process and Product Development}

\section{Seth McAfee1, Beth Buchanan', Bob Chapman'}

'Dosecann LD Inc., Charlottetown PEI, Canada, C1EOK5

The pharmacological effects of the cannabis sativa plant have been harnessed by humans for millennia across diverse applications. Traditionally, methods of delivery have focused on the inhalation of dried flower; however, in recent years there has been a growing interest in the cannabis extracts product class for inhalation, ingestion and topical application. Using a combination of analytical techniques in partnership with other leading laboratories, Dosecann (a licensed producer in Canada) has developed methods based on LC-MSMS, GC-MSMS and NMR characterization to gain a better understanding of the chemical composition of cannabis extracts throughout the extraction and purification process. With our partner laboratories, Dosecann has also been able to chemically characterize the resulting cannabis vapor for inhalation products from these extracts (i.e. vapes) and found direct correlations with the chemical composition of their liquid extract formulation. Other attributes of the cannabis vapor of inhalation products are detailed and compared to results found by others for combustible cannabis products.

\section{Clinical Reviews}

\section{Safety, Estrogenic Activity and Androgenic Activity of Herbs Commonly Used in Pregnancy}

\section{Hannah M. Paquette ${ }^{1}$, Cory S. Harris'}

University of Ottawa, Canada

Many women of the obstetric population pay great attention to their health and believe herbal medicines to be a healthier alternative to conventional medicine. Although many nurse-midwives and allied professionals support the use of herbal medicines in pregnancy, the current clinical evidence on the safety of most herbs' use in obstetric patients is scarce. Since these herbs continue to be used, further evaluation of herb safety in pregnancy is needed. As such, this study aimed to review the current literature surrounding the safety, efficacy, estrogenic activity, and androgenic activity of the commonly used herbal medicines in pregnancy and to identify the herbs that should be prioritized in future research. An open-ended search of published sources available through March 2021 was performed using OMNI and Google Scholar. This search identified herbal medicines commonly used in pregnancy and any current clinical studies surrounding their safety in pregnancy and hormonal activities. Eight of the commonly used herbal medicines in pregnancy were black cohosh, ginger, ginkgo biloba, lavender, red raspberry leaf, valerian, St. John's wort, and stinging nettle. Of these eight herbs, only one (ginger) was found to have sufficient clinical evidence of safety testing in obstetric patients. Additionally, three herbs (black cohosh, lavender, and stinging nettle) had minimal clinical evidence supporting their safe use in pregnancy and had potential for causing adverse effects. The other herbs had the potential for causing adverse effects but had moderate evidence supporting their safety. Each of the herbs had some evidence of estrogenic or androgenic activities, as well, but identified studies used different models, complicating direct comparisons. Collectively, the literature provides evidence that many of these herbs may be unsafe in pregnancy due to the potential for adverse effects and hormonal activity. Future research is needed to further characterize and quantify the hormonal activities of these herbs. 


\section{How can the Quality of Herbal/Natural Product Systematic Reviews be Improved? Vivien Rolfe \\ ${ }^{1}$ Pukka Herbs Ltd., Bengaluru, India}

Systematic reviews (SR) are valuable summaries of evidence on the effectiveness of interventions [1], and the numbers of SRs are increasing for many herbs [2]. For readers to be able to make reliable decisions from using SR, review methods need to be stringent. This paper evaluates the quality of herbal SR and makes recommendations to budding authors and journal editorial teams on what to look for in a robust review.

Quality assessments of 93 SR were obtained from two open datasets [3, 4] which used R-AMSTAR to evaluate 11 important systematic methodological steps, from search strategies to article selection and reporting [5].

The herbal SR examined varied in quality, and articles published with specialist organisations were more robust compared to those published elsewhere. Areas recommended for improvement include the minimising of bias by providing clarity on article inclusion and exclusion, and thorough searching the grey literature. It was common practice to exclude primary studies not written in English which risks omitting relevant studies. Given the indigenous and traditional origins of many herbs, this reinforces cultural inequalities that already exists in research.

[1] Hemmingway P \& Brereton N (2009). http://www.bandolier.org.uk/painres/download/whatis/Syst-review.pdf

[2] Kung, J., Chiappelli, F., Cajulis, O. O., Avezova, R., Kossan, G., Chew, L., \& Maida, C. A. (2010). The Open Dentistry Journal, 4, 84-91.

[3] Rolfe V et al. (2020). European Journal of Integrative Medicine, 40.

[4] Rolfe, Vivien (2021). R-AMSTAR quality analysis of herbal reviews. figshare. Dataset.

[5] Rolfe, Vivien (2020). S3 AMSTAR Quality Data Turmeric VRolfe 3Apr2020.xlsx. figshare. Dataset.

\section{An Overview of Systematic Reviews on Turmeric and Human Health Vivien Rolfe', Marion Mackonochie ${ }^{1}$ \\ ${ }^{1}$ Pukka Herbs Ltd., Bengaluru, India}

The clinical evidence supporting the use of Turmeric (Curcuma longa L.) in chronic disease is growing, and a wide range of treatment preparations are available $[1,2]$. This paper aims to summarise the available clinical data through completing an overview of turmeric systematic reviews (SR), with a focus on understanding which preparations are most effective.

SR were located using electronic bibliographic databases and manual searching. Article selection, data extraction and assessment of methodological quality using Revised Assessment of Multiple Systematic Reviews (R-AMSTAR) was completed by two authors $[3,4]$. The inclusion criteria included the use of turmeric/curcumin preparations taken orally for any human chronic health condition.

Overall, 65 SRs were identified covering 20 areas of health, primarily metabolic syndrome, osteoarthritis and other inflammatory conditions. Interventions included bioavailability-enhanced preparations, curcumin extracts, nanoparticles and whole turmeric powder. There was evidence supporting its use for regulating metabolic biomarkers, and for pain relief in osteoarthritis. Sub-group analyses carried out within the SR suggests that whole turmeric powder may be useful for inflammatory conditions, whereas curcumin extracts may help with metabolic syndrome. This paper will discuss the results and conclusions drawn in more detail.

[1] Rahmani AH et al. (2018). Advanced Biomedical Research, 7.

[2] Hassanzadeh K et al. (2020). International Journal of Molecular Sciences, 21(18), 6619.

[3] Mackonochie M et al. (2018). PROSPERO 2018 CRD42018115799.

[4] Shea BJ et al. (2017). BMJ 358, j4008.

\section{Natural Product Chemistry}

\section{NHP Applications of Low field NMR Chuck Chang', Sheena Kuo ${ }^{1}$ IISURA}

NHP characterization has traditionally relied primarily on UV-Visible spectroscopy and chromatography. More recently, FT-IR spectroscopy and hyphenated mass spectrometry have become more commonplace. However, few applications of NMR in this area exist even though NMR has long been known as an useful orthogonal technique. In the past, cost, space, and technical complexity have relegated applications of NMR to specialized 
research laboratories. However, recent advances have made bench-top, low-field instruments more widely available. While low field instruments cannot compete with the sensitivity, resolution and speed of high field NMR's, their ease of use and low cost of operation make them attractive in commercial applications such as routine quality control.

This presentation provides examples where low field NMR can be used as an orthogonal method of authentication for oils and herbal extracts in NHP's. Additionally, elucidation of structural information that would otherwise be unavailable through chromatography will be discussed through the case of a glucosamine polymer. Furthermore, the presentation will show how low field NMR can be useful in the case of phospholipids where quantification through HPLC is challenging.

The specific cases in this presentation make a good case for inclusion of low field NMR in a routine quality control setting. It is hoped that standardized method parameters can lead to a future common library of NMR spectra for the identification of NHP's.

Acknowledgement: The authors would like to thank Roland Gahler, Owner of Factors Group of Companies, for the financial support. ISURA is a not-for-profit independent organization.

\section{Evaluation of a Density Infrared Instrument for Ethanol Determination in Kombucha Products}

\section{Jamie Finley', Michael Chan'1, Hong Sy', Paula N. Brown ${ }^{1}$}

${ }^{1}$ British Columbia Institute of Technology, Centre for Applied Research \& Innovation, Burnaby, British Columbia, Canada

Kombucha is a fermented beverage composed of sucrose, tea and a cellulose pellicle consisting of symbiotic bacteria and yeasts. When marketed as a non-alcoholic beverage in Canada, the Food and Drug Regulations stipulate ethanol content must not exceed $1.1 \%$ alcohol by volume (ABV). A recent study by the BC Centre for Disease Control reported $31.5 \%$ of Kombucha products collected had ABV levels $>1.1 \%$ and noted that over $70 \%$ of BC processors whose products were tested potentially have problems with controlling alcohol levels. These elevated $A B V$ values were stated to be a public health threat particularly among at-risk populations including pregnant women and young children. With an identified need for processors to have access to accurate and precise methods to ensure products are safe and compliant, AOAC International issued a call for methods and established a set of Standard Method Performance Requirements (SMPR) for methods to quantify ethanol in Kombucha. Density infrared instruments are often used to measure alcoholic beverages; to establish the suitability of this approach for ethanol quantification in Kombucha, a single validation study was performed. Results indicated the instrument had acceptable precision with \%RSD ranging from $0.5-6.1 \%$ and HorRat values from $0.1-1.5$ for the 8 products tested. Accuracy of the instrument did not meet established guidelines as spike recovery analysis of samples containing $0.5,1.0$ and $1.5 \% \mathrm{ABV}$ had recoveries of $78 \%, 88 \%$ and $90 \%$. These results fall well below the $97-102 \%$ recovery range specified by the SMPR and are particularly concerning as the 0.5 and $1.0 \%$ ABV levels correspond with regulatory limits for non-alcoholic beverages in the US and Canada, respectively. This study emphasizes the need to demonstrate method fitness specifically for the intended purpose, even when using methods previously validated.

\section{Detecting Adulteration of Olive Oil Using Low Field 1H NMR Data Thomas Head' ${ }^{1}$ A. Paulina de la Mata², James J. Harynuk², Paul R. Shipley ${ }^{1}$ ${ }^{1}$ University of British Columbia, Canada \\ ¿University of Alberta, Canada}

Food items and natural health products (NHPs) are both prevalent targets of adulteration and fraud. The challenges in the detection of adulteration are similar for both NHPs and food items, the complex matrix and wide variety of metabolites makes for large datasets which are cumbersome to manually interpret.

Olive oil is a prime target for adulteration due to its price, and the relative ease which samples can be cut with lower quality olive oil or oil from other species. Authentication of products is difficult to do based on a physical examination, making instrumental analysis the primary tool for detection. An ideal method to authenticate samples would need to be cheap, robust, high throughput, and facile. Current methods of authentication utilize high field NMR instruments which can be prohibitively expensive, require highly educated users to properly operate, and 
require a large amount of infrastructure in terms of floorspace, cryogens, and sample processing. Non-NMR instruments can be similarly costly and present their own unique logistical challenges.

In our experiment, we utilize partial least squares discriminant analysis and random forest machine learning algorithms to create predictive models using low field $60 \mathrm{MHz} 1 \mathrm{H}$ NMR data to detect adulteration. Modern low field NMR instruments are cost-effective, require little training or maintenance, and fit on a benchtop. These advantages come at the cost of spectral resolution and sensitivity, making the ability to distinguish samples based on their spectra much more difficult. A proprietary spectral alignment algorithm was utilized for data processing, and the models were trained on the algorithm-aligned and manually aligned data. Using our predictive models, olive oil samples could be reliably discriminated from non-olive oil samples; additionally, the algorithm-aligned data was predicted at similar values compared to manual expert-aligned data.

\section{Trimethyl Quaternary Amine Compounds in Mushrooms}

\section{Bernd Keller ${ }^{1}$}

${ }^{1}$ Numinus Bioscience, Numinus Health \& Wellness, Canada

$\mathrm{N}, \mathrm{N}, \mathrm{N}$-trimethylated compounds play important roles in metabolic processes both in animals and plants. For example, betaines or trimethylated amino acids such as trimethyl-glycine regulate osmotic pressure in living cells. Other trimethylated compounds like cholines, are important metabolites in neurogenesis and early development. Carnitines are pivotal in transporting long-chain fatty acid into mitochondria for fatty acid oxidation and energy production. More exotic compounds like ergothioneine, found only in certain plants, mushrooms and bacteria, have gained increasing interest due to its great antioxidant activity. Many of these components are commercially available as individual supplements in the NHP market space.

Here we present some recent findings from our laboratory on trimethyl quaternary amine compounds identified in 'magic' mushrooms (Psilocybe cubensis). The presence and relatively high abundance shines new light on the nutritional and potential synergistic effects of these compounds in 'magic' but also non-psychedelic mushrooms and should be considered when formulating new supplemental or medicinal health products.

\section{Neuroscience \& Mental Health}

\section{Nootropic Activity of Three Dosage Forms of Tinospora and Glycyrrhiza in Animal Models}

\section{Swati Gadgil', Vaidehi Patankar², Priyanka Mohite², Madhuri Pawar ${ }^{1}$}

${ }^{1}$ Dept. of Rasashastra Bhaishajyakalpana, College of Ayurved, Bharati Vidyapeeth Deemed to be University, Katraj, Pune, India

¿2Self Employed Ayurved Clinician

Medhya rasayana herbs enhance cognitive functions. The herbs in specific dosage forms like pastes, fresh juice, milk treated with herb, are more effective. Stressful life, long term mental illnesses, and aging result in early decline of different cognitive functions. Three dosage forms of guduchi (Tinospora cordifolia Willd) and yashtimadhu (Glycyrrhiza glabra Linn.) are studied and compared for nootropic activity.

Guduchi juice $(20 \mathrm{ml} / 70 \mathrm{~kg})$, granules $(40 \mathrm{gm} / 70 \mathrm{~kg})$, ghee $(40 \mathrm{gm} / 70 \mathrm{~kg})$ and yashtimadhu powder with water (10 $\mathrm{gm} / 70 \mathrm{~kg})$, with cowmilk (10 gm/70 kg), milk decoction $(80 \mathrm{ml} / 70 \mathrm{~kg})$ were tested for diazepam induced amnesia using elevated plus maze in young mice orally for 15 days; and spatial learning-memory in radial arm maze in aged mice for 7 days. Diazepam $1 \mathrm{mg} / \mathrm{kg}$ was induced intraperitonially on fifteenth day and subsequent learning-memory assessment was done. In second model, animals were subjected to drug treatment with one learning trial per day for seven days, thereafter memory assessment was done on eighth day. One way ANOVA followed by Tukey's test was used.

Induction of amnesia using diazepam was confirmed. In diazepam model, all three groups of guduchi and yashtimadhu showed reduction in transfer latency on day fifteen and sixteen $(\mathrm{p}<0.001)$ except guduchi ghee $(\mathrm{p}<0.05)$ in comparison with the induction drug group. In the second model guduchi juice, and granules and yashtimadhu milk decoction showed significant increase in percentage of correct choices when compared with normal control group $(\mathrm{p}<0.05)$. Intra group comparison in case of both the herbs in both the models showed that guduchi granules and yashtimadhu milk decoction are the most effective forms. 
Guduchi granules and yashtimadhu milk decoction showed best results over other two forms. Herbs display specific action at standard dose in the particular dosage form.

\section{Efficacy of Cannabidiol Treatment for Refractory Epilepsy in Pediatrics and Potential Adverse Drug Interactions with Antiepileptic Drugs}

\section{Andie MacAndrew' ${ }^{1}$, Corrine Dobson ${ }^{1}$, Cory S. Harris ${ }^{1}$}

${ }^{1}$ University of Ottawa, Canada

This project presents and discusses currently available evidence involving the use of cannabidiol (CBD) in pediatric refractory epilepsy treatment. The adjunctive nature of this therapy provides substantial risk of adverse events. The potential for pharmacokinetic adverse drug interactions concerning CBD-anticonvulsant combinations was first identified via preclinical assessment of cytochrome P450 (CYP) enzymes involved in anticonvulsant and CBD metabolism. The effectiveness of add-on CBD therapy was then evaluated using a systematic review of literature pertaining to the safety and efficacy of treatment in pediatric clinical trials. Identified clinical evidence concerning drug interactions with specific anticonvulsant and $\mathrm{CBD}$ was used to corroborate risks regarding these interactions. FDAble adverse event reports were then assessed to identify high-risk combinations and to further evaluate drug interactions involving CBD and antiepileptic drugs in pediatrics. FDAble's database comprises data from the FDA's drug Adverse Event Reporting System. This analysis illustrates the effectiveness of CBD therapy in epilepsies previously resistant to treatment, in addition to providing a comprehensive appraisal of the potential for specific CBD-anticonvulsant drug interactions. The greatest risk for adverse interactions with CBD were found with the anticonvulsants clobazam and valproic acid. Levetiracetam was identified in a notable proportion of pediatric adverse event reports as a primary suspect but did not demonstrate evidence of an interaction with CBD in clinical data. Conversely, stiripentol was not present in identified adverse event reports but exhibited potential for interactions with CBD in clinical trials. Further studies are required to evaluate risks associated with other anticonvulsants. Altogether, results from this project indicate significant effectiveness of $\mathrm{CBD}$ as an anticonvulsant along with the corresponding risks accompanying concomitant use with other antiepileptic drugs. Subsequent research and monitoring is justified for the use of add-on CBD therapy in pediatric populations.

\section{Ashwagandha Root Extract and Ubisol-Q10 Inhibit Progression of Neurodegeneration in Alzheimer's Disease by Targeting Multiple Biochemical Mechanisms

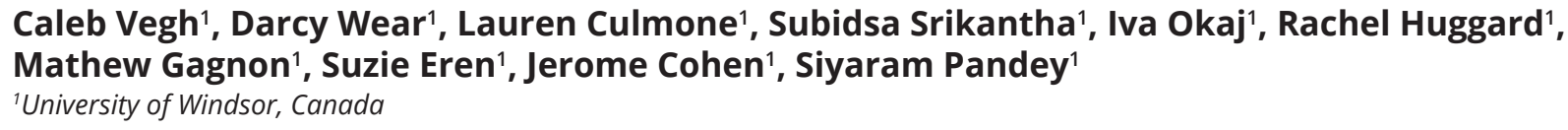

Alzheimer's Disease (AD) is the most common form of dementia and the most common neurodegenerative disease worldwide. AD is characterized by toxic beta-amyloid plaque/neurofibrillary tangle production and decline in neurocognitive function leading to severe morbidity. With an increase in the aging population, there is an unprecedented increase in the incidence of AD. There is no cure and current therapies only provide symptomatic relief, but prolonged use has shown to have toxic/adverse psychological side effects. We have found from previous research, a number of mechanisms associated with AD including oxidative stress, mitochondrial dysfunction, impaired autophagy, and inflammation. Thus, $\mathrm{AD}$ is a multifactorial disease and targeting only one mechanism may not be enough to halt $\mathrm{AD}$ neurodegeneration. Therefore, it is hypothesized that a multifactorial approach using well tolerated natural health products be used to treat AD. Ubisol-Q10, a water-soluble formulation of coenzyme-Q10, has been shown to stabilize mitochondria, inhibit oxidative stress, and enhance autophagy using in-vitro and in-vivo models of AD. Furthermore, Ubisol-Q10 reduced circulating amyloid-beta, beta-amyloid plaque formation, and memory impairment in transgenic $\mathrm{AD}$ mice. On the other hand, root extract of ashwagandha (ASH) has also been shown to be neuroprotective by reducing neuro-inflammation and beta-amyloid plaque load, and improve memory in transgenic AD mice. In order to target the multiple mechanisms of AD, we combined Ubisol-Q10 and ASH using in-vitro and in-vivo models of AD. We observed combined Ubisol-Q10 and ASH resulted in a decrease in oxidative stress, induction of autophagy, and reduced neuro-inflammation in-vitro and in-vivo. Additionally, the combination treatment reduced memory impairment and better reduced beta-amyloid plaque load in the brains of transgenic mice compared to the treatments alone. These results suggest this combination has the potential of being a well-tolerated long-term therapeutic for AD patients. 


\section{Nutriceuticals \& Functional Foods}

\section{Polyphenols Composition and Bioactive Properties of Moringa Oleifera Lam. Beverages}

\section{Ângela Fernandes ${ }^{1}$, José Pinela' ${ }^{1}$, Maria Inês Dias' ${ }^{1}$, Aducabe Bancessi², Ângela Liberal'

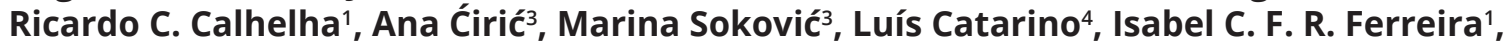 Lillian Barros ${ }^{1}$}

${ }^{1}$ Centro de Investigação de Montanha (CIMO), Instituto Politécnico de Bragança, Campus de Santa Apolónia, 5300-253 Bragança, Portugal ${ }^{2}$ Nova School of Business and Economics, NOVA University of Lisbon, Campus de Carcavelos, Rua da Holanda, n. 1, 2775-405 Carcavelos, Portugal; and Centre for Ecology, Evolution and Environmental Changes (CE3c), Faculty of Sciences, University of Lisbon, Campo Grande, $1749-016$ Lisbon, Portugal ${ }^{3}$ Institute for Biological Research "Siniša Stanković" - National Institute of Republic of Serbia, University of Belgrade, Bulevar Despota Stefana 142, 11000 Belgrade, Serbia

${ }^{4}$ Centre for Ecology, Evolution and Environmental Changes (CE3c), Faculty of Sciences, University of Lisbon, Campo Grande, 1749-016 Lisbon, Portugal

Moringa (Moringa oleifera Lam.) is a fast-growing deciduous tree whose leaves, flowers, fruits and seeds have been used as a sustainable alternative for preventing and alleviating/relieve malnutrition in many tropical countries, where it is known as "tree of life" due to its medicinal properties and health-promoting effects. Given the multiple applications of moringa as food and medicine, this study was carried out to provide a detailed characterization of bioactive constituents and properties of herbal beverages prepared with seeds, flowers and fruits of this plant. The samples were collected in Guinea-Bissau and prepared in infusions and decoctions, following traditional recipes of folk medicine, and hydroethanolic extracts. The phenolic profiles were analysed by HPLC-DAD-ESI/MS. Antioxidant, anti-inflammatory and cytotoxic activities were evaluated in vitro using different cell-based assays and antimicrobial effects were screened against food-borne fungi and bacteria by serial microdilution methods. Flavonoids were the most abundant group of phenolic compounds identified in the moringa samples, with glycosylated derivatives of quercetin having a numerical expression superior to other flavonoid aglycones. In general, hydroethanolic extracts contained more phenolic compounds and were more active against lipid peroxidation, nitric oxide production, and tumour cells growth. Antimicrobial effects against the tested microorganisms were displayed by both hydroethanolic and aqueous extracts. These results highlighted the biological properties of moringa preparations, thus validating the functional effects of these beverages.

Acknowledgments: FCT, Portugal, for financial support through national funds FCT/MCTES to CIMO (UIDB/00690/2020), to cE3c (UIDB/00329/2020), and to the A. Bancessi grant (SFRH/BD/135356/2017). National funding by FCT, P.I through the institutional/individual scientific employment program-contract for A. Fernandes, J. Pinela, M.I. Dias, R.C. Calhelha, and L. Barros contracts. To FEDER-Interreg España-Portugal programme for financial support through the project TRANSCoLAB 0612_TRANS_CO_LAB_2_P. Also to the Ministry of Education, Science and Technological Development of the Republic of Serbia (451-03-68/2020-14/200007).

\section{Development of Natural Hypocholesterolemic Agents: Application in Cottage Cheese Filipa A. Fernandes ${ }^{1,2}$, Dora Khouja', Márcio Carocho', Ricardo Calhelha', Bruno Melgar1, Paula Rodrigues ${ }^{1}$, Filipa Reis', Sandrina A. Heleno', Isabel C. F. R. Ferreira', Lillian Barros ${ }^{1}$ \\ ${ }^{1}$ Centro de Investigação de Montanha (CIMO), Instituto Politécnico de Bragança, Campus de Santa Apolónia, 5300-253 Bragança, Portugal ${ }^{2}$ Grupo de Nutrición y Bromatología, Departamento de Química Analítica y Alimentaria, Facultad de Ciencias de Ourense, Universidad de Vigo-Ourense Campus, E-32004 Ourense, Spain}

This work aimed at exploiting mushroom bioresidues, namely Agaricus bisporus L. to obtain mycosterols and develop hypocholesterolemic functional foods. Mycosterols enriched extracts were obtained by ultrasound assisted extraction, characterized in terms of mycosterols through HPLC-UV, evaluated for their toxicity in non-tumor cells (PLP2) and hypocholesterolemic capacity using a $\mathrm{CaCo}_{2}$ cell line. After incorporation of the active dose in cottage cheeses, and to evaluate the incorporation effects and the bioactivity maintenance, the nutritional value of the developed cheeses was evaluated, as also the physical parameters, and the microbial load over a shelf life of 9 days. The color of the cheeses incorporated with the extract was the brownest compared to the other two samples (cottage cheese with ergosterol and control cottage cheese). Palmitic was the prevalent fatty acid, followed by oleic and capric acid, being the saturated fatty acids the major ones. Lactose and glucose were the two found soluble sugars; being glucose observed only in cheese incorporated with A. bisporus. Also, the incorporations did not cause any significant alterations to the normal flora found in the cottage cheese. 
The cheese with pure ergosterol and the cheese incorporated with A. bisporus extract reduced cholesterol absorption by $21.1 \%$, and $30.24 \%$, respectively, thus validating thehypocholesterolemic potential of mycosterols while promoting sustainability through the use of food waste/by-products.

Acknowledgments: Foundation for Science and Technology (FCT, Portugal) for financial support through national funds FCT/MCTES to CIMO (UIDB/00690/2020) and F.A. Fernandes PhD grant ((SFRH/BD/145467/2019). L. Barros and C. Calhelha also thank the national funding by FCT, P.I., through the institutional scientific employment program-contract for their contracts and, M. Carocho and S.A. Heleno to the national funding by FCT, P.I., through the individual scientific employment program-contracts. European Regional Development Fund (ERDF) through the Regional Operational Program North 2020, within the scope of Project Mobilizador Norte-01-0247FEDER-024479: ValorNatural ${ }^{\circledR}$.

\title{
Improvement of Bakery Products Functional Properties with the use of Non-Conventional Seeds Flour
}

\author{
Juliana França Lima1, Maria Inês Dias², Tiane C. Finimundy², João C.M. Barreira², Marija Ivanov³, \\ Marina Sokovic ${ }^{3}$, Nádia Cristiane Steinmacher ${ }^{4}$, Isabel C. F. R. Ferreira², Lillian Barros² \\ ${ }^{1}$ Investigação de Montanha (CIMO), Instituto Politécnico de Bragança, Campus de Santa Apolónia, 5300-253 Bragança, Portugal. Departamento \\ Acadêmico de Alimentos (DAALM), Universidade Tecnológica Federal do Paraná, Campus Medianeira, 85884-000, Paraná, Brasil \\ 2Investigação de Montanha (CIMO), Instituto Politécnico de Bragança, Campus de Santa Apolónia, 5300-253 Bragança, Portugal \\ 3Institute for Biological Research "Siniša Stanković" - National Institute of Republic of Serbia, University of Belgrade, Bulevar despota Stefana 142, \\ 11000 Belgrade, Serbia \\ ${ }^{4}$ Departamento Acadêmico de Alimentos (DAALM), Universidade Tecnológica Federal do Paraná, Campus Medianeira, 85884-000, Paraná, Brasil
}

The flours obtained from the seeds of niger, millet, and birdssed, unconventional food plants (PANC) $[1,2]$, were assessed regarding physical and nutritional characteristics (AOAC methods). The bioactive properties (antioxidant, antimicrobial) of their hydroethanolic extracts were also assessed. Finally, bread samples were prepared with partial replacement of the wheat flour (20\% of PANC's flour) to compare the final characteristics of the breads. The three flours presented high granulometry, associated with a high-water absorption index, indicating a necessary complementation with other flours for bakery application. Niger seed stood out with the best nutritional profile, and its extracts presented the highest antioxidant and antimicrobial properties. Niger and millet presented a strong antifungal capacity (greater than the positive controls). None of the samples presented hepatotoxicity. The breads obtained with millet and birdseed flour presented a similar behavior to the control samples (100\% wheat flour) regarding the texture, specific volume, and color. This study proved the beneficial contribution that PANC flours can bring for the development of new bakery products.

[1] Kinupp, V.F., \& Barros, I.B.I. Horticultura brasileira v. 22(2004), 17-25.

[2] Leal, M.L. (2015). Repositório Institucional UFSC. https://repositorio.ufsc.br/handle/123456789/174789

Acknowledgments: The authors are grateful to the Foundation for Science and Technology (FCT, Portugal) for financial support through national funds FCT/MCTES to CIMO (UIDB/00690/2020); national funding by FCT, P.I.: institutional (L. Barros; M.I. Dias; C. Pereira) and individual (J.C.M. Barreira) scientific employment program-contract; FEDER-Interreg España-Portugal programme (TRANSCoLAB 0612_TRANS_CO_LAB_2_P). EERDF: Regional Operational Program North 2020, GreenHealth - Digital strategies in biological assets to improve well-being and promote green health, Norte-01-0145-FEDER-000042; Ministry of Education, Science and Technological Development of Republic of Serbia (451-03-68/2020-14/200007).

\section{Mini Review on Meat Based Functional Food}

\section{Sarika Patil ${ }^{1}$, Asmita Wele ${ }^{1}$}

${ }^{1}$ Bharati Vidyapeeth College of Ayurveda, Department of Rasashastra and Bhaishajyakalpana (Ayurveda Pharmacology), Pune, India

Dietary supplements, nutraceuticals, and functional foods are the terms, that are frequently used interchangeably. However functional food is food, which can be consumed as a part of a regular dietary regimen and not merely a capsule, tablet, or pill. The use of functional food has gained momentum in today's world with the development in the health sector. In this COVID-19 pandemic, the perception of health and nutrition has turned people more towards the natural means of regulating health including food. Nutritional science has its roots in ancient Ayurvedic practices which date back to the 12th century BC. Food is praised to be superior to all medicines (Mahabheshaja) as it is essential in both health and disease equally. Sick persons cannot survive on medicines alone, they need food. 
Functional foods are nature-derived dietary articles that offer satiety, provide the essential nutrients, deliver certain therapeutic benefits, and improve the quality of life. Traditionally, Ayurvedic texts advise infusing foods with spices, oils, essences, flavors, and colors. Further, they are subjected to specific procedures, which enhance the nutritional value as well as add the therapeutic effect.

Out of various food groups, meat is an excellent source of protein loaded with vitamins and minerals required for normal body functions. Meat though in regular use worldwide, it's overuse is known for the unintended effects on health. Ayurvedic pharmacopeial literature (Nighantu grantha) gives the properties and utility of meat of a vast variety of animals and birds. The use of meat products in specific conditions like debility, pregnancy and to acquire bodybuilding is addressed. The present mini literature review focuses on the use of meat from different sources, their characteristics, pharmacological effects, and traditional recipes. It also deals with contemporary techniques of conversion of meat into functional foods.

\section{Can a Bitter Herb Found in Beer Reduce Inflammation?}

\section{Ryan Pusiak ${ }^{1}$, Cory S. Harris ${ }^{1}$}

${ }^{1}$ Department of Biology, University of Ottawa, Canada

Hops, Humulus lupulus, flowers are primarily cultivated for the production of beer with hundreds of registered cultivars that vary in chemistry and flavour. Hops have also been used in natural health products for its anxiolytic, sedative, and estrogenic properties. The anti-inflammatory properties of hops remain understudied as research has primarily focused on only a few isolated pure compounds from hops. The cyclooxygenase (COX) family of enzymes mediate prostanoid production and inflammation, particularly COX-2, which is inducible along the inflammatory cascade. Whereas humulone (alpha-acid) and xanthohumol (prenylated chalconoid) from hops reportedly inhibit COX-2 activity at low $\mu \mathrm{M}$ levels hop flowers contain a plethora of other compounds that might inhibit COX-2. Few studies have tested the effects of hop flower extract on COX-2 inhibition and no study has compared the activity of multiple cultivars. Therefore, this study investigated 35 hop cultivar extracts (46 total samples) for COX-2 inhibition and subsequent biochemometric analyses. The $\mathrm{IC}_{50}$ across all samples was $7.03 \mu \mathrm{g} / \mathrm{ml}(\mathrm{SE}=0.29,6.45-7.61)$. I also tested major marker compounds and found that xanthohumol $\left(\mathrm{IC}_{50}=17.5 \mu \mathrm{g} / \mathrm{ml}, 49.4 \mu \mathrm{M}\right)$, Ad-humulone $\left(\mathrm{IC}_{50}=\right.$ $1.74 ; 4.8 \mu \mathrm{M})$, and Ad-lupulone $\left(\mathrm{IC}_{50}=2.42 \mathrm{ug} / \mathrm{ml} ; 5.8 \mu \mathrm{M}\right)$. Following phytochemical analysis by High Performance Liquid Chromatography (HPLC-DAD), I analyzed COX-2 inhibition relative to chemistry and found that none of the tested compounds predicted the COX-2 inhibition. However, with $\mathrm{IC}_{50}$ values for extracts at comparable concentrations, the levels of marker compounds in extracts were insufficient to contribute to activity, indicating that other metabolites are driving COX-2 inhibition. Future metabolomics work may help identify and characterize these overlooked bioactive compounds in hops.

\section{Pharmacology \& Toxicology}

\section{A Natural Product Isolated from a Canadian Prairie Plant Induces a Cellular Effect that Mimics those in Patients Afflicted with Rare Laminopathy Diseases Chad R. Beck ${ }^{1}$, Jan M. Tuescher ${ }^{1}$, Benjamin Yeremy ${ }^{2}$, Raymond J. Andersen ${ }^{1}$, Roy M. Golsteyn ${ }^{1}$ \\ ${ }^{1}$ University of Lethbridge, Canada \\ 2University of British Columbia, Canada}

The scientific and medicinal potential of natural products isolated from Canadian prairie plants is relatively unexplored. In the Prairie to Pharmacy Program, we use an unbiased phenotypic (imaging) assay to investigate extracts prepared from prairie plants. Extracts PP-630 prepared from the plant Symphoricarpos occidentalis (Caprifoliaceae) induces nuclear-associated vacuoles (NAVs) in human cells. NAVs are clear vacuoles adjacent to the nucleus. Such vacuoles are rare in cells, but NAVs are caused by mutations of the lamin B receptor (LBR) gene in the human rare genetic diseases known as laminopathies, Greenberg skeletal dysplasia and Pelger-Huët anomaly. By biology-guided fractionation, we isolated pheophorbide $\mathrm{A}(\mathrm{PhA})$ and identified conditions to induce NAVs by $\mathrm{PhA}$ and light treatment in human U2OS cells. By transmission electron microscopy, we observed separation of the double nuclear membrane in PhA treated cells, similar to NAVs from LBR mutants. Plasmids encoding mutated LBR acquired from Dr. H. Herrmann were transfected into U2OS cells, and a second culture was treated with $\mathrm{PhA}$. Each produced similar results as determined by light microscopy. The nuclear pore complex and lamin A/C proteins were investigated in transfected 
mutants and PhA treated cells by immunofluorescence microscopy. Each protein was similarly distributed around the nuclear membranes in PhA or LBR mutant induced NAVs. These results suggest that NAVs formed by PhA treatment mimic those induced by Greenberg skeletal dysplasia and Pelger-Huët anomaly. This report of chemically induced NAVs demonstrates the biological potential of natural products for study of cell biology and human diseases.

\title{
Egg White-Derived Bioactive Peptide Effect on Plasma Biochemical Parameters and Liver Lipid Accumulation of High Fat Diet Induced Obese and Insulin Resistant Mice Stepheny Carneiro de Campos Zani', Ren Wang', Jianping Wu', Catherine B. Chan'1 ${ }^{1}$ University of Alberta, Canada
}

Ectopic lipid accumulation in peripheral non adipose tissues is linked with insulin resistance (IR), obesity and metabolic disorders. An egg white-derived peptide (PEP2) was previously shown to enhance PPAR $\gamma$ protein abundance in adipocytes. Moreover, PEP2 is present in a mixture of egg peptides shown previously to improve glucose tolerance and insulin sensitivity in rats. Given the role of PPAR $\gamma$ in lipid metabolism and glucose homeostasis, we aimed to investigate the effect of PEP2 on liver lipid accumulation and plasma biochemical parameters. C57BL/6 mice were fed high fat diet (HFD) for 6 weeks to induce obesity and IR. Mice were then divided into 3 groups, HFD, HFD+PEP2 (45 $\mathrm{mg} / \mathrm{Kg} \mathrm{BW}$ ) and HFD+ROSI (rosiglitazone $2.5 \mathrm{ug} / \mathrm{Kg} \mathrm{BW}$ ) and received their diets for another 8 weeks. One group received low fat diet (LFD) throughout the 14-week period. Plasma biochemical parameters were measured by ELISA and colorimetric assays, and liver paraffin blocks and tissue slides were prepared for histological analysis (hematoxylin \& eosin staining, 20× magnification, ImageJ software). PEP2, but not ROSI, partially normalized fasting blood glucose compared to HFD. Plasma lipid profile showed no statistical changes despite a reduction in triglycerides in all groups compared to HFD. PPAR $\gamma$ protein abundance was increased by HFD feeding. Interestingly, ROSI increased liver weight and triglycerides accumulation compared to the other groups. Morphological characterization showed that PEP2 presented lower inflammatory infiltration than HFD, smaller lipid droplet (LD) size compared to HFD and ROSI, and more cells per area than ROSI. In conclusion, despite no major changes in plasma parameters among the groups, ROSI exacerbated liver lipid accumulation induced by HFD while PEP2 prevented the formation of macro LD, attenuated hepatocyte hypertrophy and hepatic inflammation, thus presenting similar liver morphology to LFD.

\section{Exposure of Bacteria to Prairie Plant Extracts Induces Unique Clump Phenotype with Prospective Applications}

\author{
Nadia Hand ${ }^{1,2}$, Tianna Gerber ${ }^{1}$, Kaitlyn Grisnich ${ }^{1}$, Leanne DuMontier ${ }^{1}$, Sophie Kernéis ${ }^{1}$ \\ ${ }^{1}$ Microbial Research Laboratory, Lethbridge College, Canada \\ ${ }^{2}$ University of Lethbridge, Research Internship Concentration, Canada
}

Since 2017, the World Health Organization (WHO) has declared antibiotic resistance to be a topic of high health priority, commenting that if no action is taken we might return to an era when people are at great risk of dying of common infections. One recommendation is to discover new antibiotics or alternative methods to control bacterial infection. Current antibiotics have been isolated solely from fungi and bacteria while none have plant origins despite the presence of traditional plant medicines with antibacterial uses. This study focused on the identification of plants native to Alberta with antibiotic properties and observed that some plant extracts have a unique "bacterial clumped" phenotype. Staphylococcus epidermidis was used to characterize the clumping activity of the plant extract NP856 from the Polygonaceae family. We found that the time to produce clumps is dependent on bacterial concentration with clumping occurring as early as 5 minutes when using an overnight culture of S. epidermidis. S. epidermidis in the clumps are still alive, and the plant extract clumped more than $90 \%$ of the bacteria in suspension. Bacterial clumps are not a biofilm, as NP856 can clump bacteria fixed in glutaraldehyde, which is inconsistent with the characteristic of biofilms that require bacteria-produced exopolysaccharide matrix. This clumping activity observed for NP856 may have applications in limiting bacterial infection by removing them from liquid sources, such as clearing bacterial contaminants from water found in large animal farm facilities. Future studies will incorporate other bacteria such as E. coli and S. aureus, major contaminants in agriculture and in cosmetics industries to extend the potential application of this new characterized phenotype. 


\title{
Characterization of the Antibacterial Activity of Aponogeton Madagascariensis Crude Anthocyanin Extracts
}

\author{
Michaela Kember ${ }^{1}$, Zhenyu Cheng ${ }^{2}$, Arunika N. Gunawardena ${ }^{3}$ \\ ${ }^{1}$ Biology Department, Faculty of Science, Dalhousie University \& Microbiology and Immunology, Faculty of Medicine, Dalhousie University, Canada \\ ${ }^{2}$ Microbiology and Immunology, Faculty of Medicine, Dalhousie University, Canada \\ ${ }^{3}$ Biology department, Faculty of Science, Dalhousie University, Canada
}

The phenomenon of antibiotic resistance represents one of the most concerning threats to global healthcare systems worldwide. Plant preparations used in traditional medicine against bacterial infections have been found to inhibit growth of various bacterial species and may represent a promising potential source of natural bioactive compounds. Anthocyanins are a type of flavonoid that have demonstrated antibacterial activity against Gram positive bacteria. The lace plant (Aponogeton madagascarensis) belongs to the family Aponogetonaceae, which is well known in traditional medicine and additionally displays unique developmental morphology. The unidentified bioactive anthocyanins present in the lace plant represent uncharacterized potential for medicinal application. The goal of this investigation was to determine if crude anthocyanin extracts from lace plant leaves had antibacterial efficacy against Gram positive bacteria, and if so, the mode of antibacterial action that the compounds had against the bacterial species. In this study, the crude anthocyanin extracts taken from two stages of lace plant leaf development were applied at four different concentrations to three bacterial species including Enterococcus faecalis, methicillinresistant Staphylococcus aureus, and Listeria monocytogenes. The crude anthocyanins displayed selective inhibition of the growth of the selected bacterial species: E. faecalis displayed the most sensitivity to the anthocyanin treatments in a concentration-dependent manner, L. monocytogenes displayed moderate sensitivity, and S. aureus demonstrated resistance to treatment. To investigate the effects of the anthocyanins on the bacterial species, treated bacteria were imaged using transmission electron microscopy (TEM). TEM analysis revealed that E. faecalis cells hadcell wall damage including apoptotic morphologies, inappropriate septa formation, and an inability to fully separate. These results suggest that the primary mode of action of the anthocyanins may be interaction with the bacterial cell wall, inhibiting cell replication. The selective inhibition of the bacterial species selected here by the crude extracts is deserving of further investigation.

\section{Evaluation of In Vitro Neutralizing Effects of Aqueous Extract of Pogostemon Heyneanus Leaves and its Fractions}

Rathnasekara H. L. ${ }^{1}$, Jayasuriya W. J. A. B. N. ${ }^{1}$, Arawwawala L. D. A. M. ${ }^{2}$, Suresh T. S. ${ }^{3}$

${ }^{1}$ Department of Pharmacy and Pharmaceutical Sciences, Faculty of Allied Health Sciences, University of Sri Jayewardenepura., Sri Lanka

${ }^{2}$ Herbal Technology Section, R \& D Complex, Industrial Technology Institute, Halbarawa, Thalahena, Malabe, Sri Lanka

${ }^{3}$ Department of Biochemistry, Faculty of Medical Sciences, University of Sri Jayewardenepura., Sri Lanka

Pogostemon heyneanus Benth. (Indian Patchouli or Kollan kola) is an undershrub herb of the Indian subcontinent which belongs to the family Lamiaceae and is used as a remedy for chronic dyspepsia. This study investigates the antacid potential of different concentrations of aqueous extract (AE) of $\mathrm{P}$. heyneanus leaves, its ethyl acetate fraction (EAF) and residual aqueous fraction (RAF) using in vitro methods. The end $\mathrm{pH}$ of each extract and their neutralizing effect on artificial gastric acid (AGA) were determined and compared with distilled water and standard drug, ENO. The neutralizing capacity was determined using the titration method of Fordtran. A modified model of Vatier's artificial stomach was used to determine the duration of consistent neutralization of AGA. All the tests were run in triplicate. Statistical analysis was performed using SPSS 25.0. End $\mathrm{pH}<3$ was considered to have no neutralizing effect. Powder microscopy and detailed anatomical characteristics of P. heyneanus were examined. Qualitative analysis of phytochemicals were performed by thin layer chromatography. Different concentrations of AE (18.6 and $37.2 \mathrm{mg} / \mathrm{ml})$ and RAF $(21.5$ and $43.0 \mathrm{mg} / \mathrm{ml})$ demonstrated a significant $(\mathrm{p}<0.001)$ neutralizing effect. RAF at a concentration of $43.0 \mathrm{mg} / \mathrm{mL}$ exhibited the highest neutralizing capacity and a higher duration of consistent neutralization $(\mathrm{p}<0.001)$ among the tested doses. EAF was found to have no neutralizing effect. Fibers, sclereids and trichomes were found in relatively high abundance in the powdered samples and dicotyledon cell organization was observed. Phenolics, flavonoids, tannins, alkaloids, saponins, terpenoids and cardiac glycosides were present in both AE and RAF and steroids were not present. Total polyphenol and flavonoid content of AE were $0.45 \mathrm{mg} / \mathrm{g}$ and $1.34 \mathrm{mg} / \mathrm{g}$ respectively. In conclusion, $\mathrm{AE}$ and $\mathrm{RF}$ exhibited a potent antacid effect in both models when determining neutralizing capacity. Retention of the antacid potential in the RAF suggests that the compounds responsible for the activity are polar. 


\section{Modeling Echinacea Alkylamide Interactions with Cannabinoid Receptors}

Tori K. Scherle', Gabriel Guillet', Cory S. Harris ${ }^{1}$

${ }^{1}$ Department of Biology, University of Ottawa, Ottawa, ON, Canada K1N

Alkylamides are a class of phytochemicals found in the roots and aerial parts of plants of the Echinacea genus, as well as other plant taxa. While alkylamides are diverse in structure and bioactivity, certain alkyamides are structurally similar to anandamide, an endocannabinoid, and are known to interact with cannabinoid (CB) receptors. In this study the relative affinities of 33 alkylamides, anandamide, cannabidiol (CBD) and $\Delta$-9-tetrahydrocannabinol (THC) for human $\mathrm{CB} 1$ and $\mathrm{CB} 2$ receptors were modelled and compared. The active sites and molecular interactions were assessed for similarities. Relative affinities between alkylamides and CB receptors were assed using Autodock Vina computer docking software in the PyRx/Python suite and binding site realization was accomplished using PyMol computer modeling software. Most alkylamides with the highest affinities for $\mathrm{CB} 1$ and $\mathrm{CB} 2$ receptor bind in the same binding pocket on each receptor but, despite previous predictions, the residue interactions vary. Furthermore, no pattern was established for predicting relative affinity based on ligand structure but the affinity of most alkylamides and both THC and CBD were higher than that of anandamide. Comparison to experimental CB receptor binding data revealed a moderate correlation between affinities from Autodock Vina and Ki values determined in vitro. This study serves as a base for future use of docking software in assessing new potential Natural Health Products and understanding the interactions of alkylamides with $\mathrm{CB} 1$ and $\mathrm{CB} 2$ receptors.

\section{(Psychedelics) Policy \& Regulation}

\section{Strides in Natural and Traditional Medicines When They Are Controlled Substances; Navigating Regulations that Govern Psychedelic Plants and Fungi}

\section{Sharan Sidhu ${ }^{1}$}

${ }^{1}$ Numinus Bioscience, Numinus Health \& Wellness, Canada

Psychotria viridis, Psilocybe species and Lophophora williamsii are all species that contain constituents that are psychedelic in nature and controlled in Canada under the Controlled Drugs and Substances Act. These plants and fungi also have a history of traditional and cultural use and present unique therapeutic and treatment opportunities in modern western medicine which has recently gained momentum and interest in the mainstream medical community. With a shift in treatment paradigms and the need to develop more effective tools for various mental health conditions, these medicinal plants are being studied more intently both at the bench top level and in clinical trials. Currently, traditional herbal medicines are typically regulated as Natural Health Products where traditional health claims can be made for self care products and not Schedule A health claim, as mental health conditions such as treatment resistant depression and PTSD would be classified. This presents regulatory challenges as well as product development challenges as certain botanical/fungal materials and associated constituents are controlled. Further, natural extracts present additional challenges for regulatory approval for supervised treatment due to inherent variability that could present challenges on meeting label claims for dosing. Here we present an overview of the regulations that govern the constituents and the botanical/fungal materials and how these may be navigated to develop products for psychedelic assisted psychotherapy and other suitable scheduled conditions. This also presents an opportunity to look at the state of traditional medicine as it converges with modern medicine around psychedelic substances.

\section{Seeking A Clear Path Forward: A Proposal for the Creation of a Traditional Medicine Containing a Controlled Psychoactive Substance Regulation for Medical or Spiritual/ Religious Use under the Food and Drug Act}

\section{Jennifer Tobe ${ }^{1}$ \\ ${ }^{1}$ M. Jennifer Tobe Law Corporation}

This paper proposes the creation of a novel regulation under the Food and Drug Act (FDA) for Traditional Medicines containing a controlled psychoactive substance (TMCPS). TMCPS include: psilocybin mushrooms, cacti containing mescaline, ayahuasca, iboga, coca leaves, and opium poppy (to name a few). Increasing demand for legal access to TMCPS for medical or spiritual/religious purposes, pose questions around current drug policy. 
The Health Canada pathway for licensing natural health products used as Traditional Medicines under the FDA Natural Health Products Regulations, provides a good regulatory model for the creation of a TMCPS regulation that can be built upon with added controls. TMCPS regulatory requirements and guidance documents could address historical and current usages of the particular TMCPS, clinical trials for new usages of TMCPS, requirements for Canadian licensed producers, government support of institutional researchers focusing on ongoing public health and safety studies, medical use prescriptions, spiritual/religious use access, cultivation and possession for personal medical or spiritual/religious use, and import/export, as well as other issues. Moreover, access through a regulated supply of TMCPS could reduce health and safety concerns created by the expanding illicit trade and minimize the increasing special exemption applications to Health Canada for access to TMCPS for research, medical, and spiritual/religious purposes.

Documentaries, news stories, and publications covering clinical trials, historical usages, retreats, opioid treatment, and personal attestations of the medical or spiritual/religious benefits, combined with the popularity of travel to countries where medical access or spiritual/religious experiences are more readily available, further validate growing interest in and demand for TMCPS.

\section{Establishing an Evidence-Based Compassionate Access Program for Psilocybin-Assisted Psychotherapy in Canada}

\section{Evan Wood ${ }^{1}$, Sharan Sidhu' ${ }^{1}$, Devon Christie M. D. ${ }^{1}$, Gabor Maté1, Lindsay MacKay', Elena Argento' ${ }^{1}$ Numinus Health \& Wellnesss, Canada}

There is a large and growing burden of serious mental health conditions affecting many Canadians. However, existing treatments are known to often be ineffective. In this context, a substantial body of research, including the completion of a growing number of randomized clinical trials, has highlighted psilocybin-assisted psychotherapy as an emerging and novel approach for the treatment of a host of mental health conditions, including treatment-resistant depression, substance-use disorder, and severe anxiety associated with a terminal diagnosis. Different cultures worldwide have used Psilocybe mushrooms in religious and healing ceremonies for thousands of years. Specifically, Psilocybe mushrooms have a long history in traditional medicinal use, with documented use going back to the 16th century by Indigenous communities in Mexico. While certain companies are developing synthetic versions of psilocybin, other organizations have Health Canada approval to research psilocybin mushroom extractions and formulations not dissimilar to other naturally derived health products. All of this is occurring in the context of widespread international interest to increase patient access to psilocybin-assisted psychotherapy given the impressive and growing track records of safety and efficacy. For instance, in November 2020, Oregon became the first U.S. state to vote to create a regulated system for access to psilocybin-assisted psychotherapy. Other U.S. and international jurisdictions are similarly looking at "right to try" legislation and other mechanisms to allow access to psilocybin-assisted psychotherapy. This presentation will outline research and regulatory considerations and argue that Health Canada should move quickly to establish a new regulatory regime for the safe and evidence-based access to psilocybin assisted psychotherapy.

\section{Product Innovations}

\section{Understanding Future Contributors: Creating a Database of Natural Health Product Researchers to Survey their Attitudes Towards Publishing and a New Field-Specific Journal}

Jeremy Y. Ng1', Halton Quach¹, Ming Zheng1', Tushar Dhawan'1, Julian Vincent T. Dychiao', Aisha Hashmani, Bismah Jameel ${ }^{1}$, Kirrthana Jegathesann', Leah Kogan' ${ }^{1}$, Xiao Wen (Vivian) Li ${ }^{1}$, Natasha Reyes' ${ }^{1}$, Jill Shah', Fred Ashbury², Kieran Cooley ${ }^{3}$, Pierre S. Haddad ${ }^{4}$ ${ }^{1}$ Department of Health Research Methods, Evidence, and Impact, Faculty of Health Sciences, McMaster University, Hamilton, Ontario, Canada 2Institute of Health Policy, Management and Evaluation, University of Toronto, Toronto, Ontario, Canada ${ }^{3}$ Department of Research, Canadian College of Naturopathic Medicine, Toronto, Ontario, Canada

${ }^{4}$ Department of Pharmacology and Physiology, Faculty of Medicine, University of Montreal, Montreal, Quebec, Canada

The distinction between a new, legitimate versus a predatory publisher is often difficult to discern given their many similarities, which can result in confusion for researchers. In this study, the creation of a database of natural health product (NHP) researchers with the intent of surveying their opinions and preferences on open access publishing and a new 
field-specific journal is described. An NHP researcher database was created including the regions of Canada, United States, West Europe and Australia, to reflect the major English-speaking regions of the world. Keyword searches of publically available data online, including faculty webpages and publications, populated the database. Duplicates were removed and saturation was reached. Researcher name, affiliation, academic rank, research interest, region and faculty webpage URL were collected. All database information was verified or corrected by independent review. Searches yielded 1894 NHP researchers: USA $(\mathrm{n}=981,51.8 \%)$, West Europe $(\mathrm{n}=337,17.8 \%)$, Australia $(\mathrm{n}=336,17.7 \%)$, and Canada $(n=240,12.6 \%)$. The large majority of researchers either had a research interest or published research relating to the traditional use, phytochemical composition, and therapeutic properties of NHPs ( $\mathrm{n}=1238,65.4 \%)$, followed by NHPs in clinical settings and healthcare education $(n=519,27.4 \%)$. Other research interests also included, but were not limited to, NHP cultivation/harvest, industrial innovations, manufacturing, and regulatory affairs. Completion of the database has created a structured participant pool for the forthcoming survey. Recruitment of additional participants is planned via snowball sampling by inviting all researchers in the database to recommend colleagues from any region of the world. It is hoped that the findings will help contributors discern the legitimacy of the journal, and that this process of stakeholder identification and engagement will benefit other academics interested in publishing or journal creation.

\section{Bio-Residues of Ilex Paraguariensis A. St. Hil. as a Source of Phenolic Compounds with Preservative Potential}

Eliana Pereira ${ }^{1}$ Bárbara Mernezes ${ }^{1}$, Cristina Caleja ${ }^{2}$, Tânia Pires ${ }^{1}$, Oludemi Taofiq ${ }^{1}$, Ricardo Calhelha ${ }^{1}$, José Pinela', Marina Sokovic ${ }^{1}$, Fernanda Vitória Leimann ${ }^{3}$, Isabel C. F. R. Ferreira², Lillian Barros ${ }^{1}$

${ }^{1}$ Centro de Investigação de Montanha (CIMO), Instituto Politécnico de Bragança, Campus de Santa Apolónia, $5300-253$ Bragança, Portugal

${ }^{2}$ Post-Graduation Program of Food Technology (PPGTA), Federal University of Technology - Paraná - UTFPR, Campus Campo Mourão, via Rosalina Maria dos Santos, 1233, CEP 87301-899, Campo Mourão, PR, Brazil

3Institute for Biological Research «Siniša Stanković» - National Institute of Republic of Serbia, University of Belgrade, Bulevar Despota Stefana 142, 11000 Belgrade, Serbia

The use of Agro-industrial wastes translates into an advantageous opportunity for the development of by-products [1]. Ilex paraguariensis A. ST. HIL. (yerba mate) is a tree widely cultivated and appreciated with a chemical composition that identifies several beneficial properties to the human body [2]. In this work, a comparison between the extracts of dehydrated yerba mate (EMNP) and bio-waste (EMP) leaves from the yerba mate industry in Brazil was made, as well as the incorporation of the EMP extract in a traditional food product, to evaluate its preservative potential. The individual profile of phenolic compounds was determined by HPLC-DAD-ESI/MS and the bioactive potential was assessed through in vitro tests for antioxidant, anti-inflammatory and antimicrobial activity, as well as cytotoxicity tests, in human tumor cell lines and in a non-tumor cell line. Among the seven phenolic compounds identified, four were found in greater quantity in the extracts of bio-residues of yerba mate (EMP). Regarding the functional properties, the extracts obtained revealed a high antimicrobial potential against the tested strains, as well as a very promising antioxidant and anti-inflammatory activities, without toxicity. After incorporating the most promising extract, EMP, in pancakes, the centesimal evaluation and the chemical composition (individual profile of sugars and fatty acids) revealed that after storage for 3 days the natural ingredient proved to show preservative efficiency.

This study intended to develop new solutions that allow reduced application of artificial additives in food products.

[1] Toop, T.A., Ward S., et al. (2017). Energy Procedia, 123, 76-80.

[2] Vieira, M.A., Rovaris, A.A., et al. (2008). Journal of agricultural and food chemistry, 56, 4637-4642.

Acknowledgments: The authors are grateful to FCT, Portugal for financial support by national funds FCT/MCTES to CIMO (UIDB/00690/2020). The authors are also grateful to FEDER-Interreg España-Portugal programme for financial support through the project the 0612_TRANS_CO_LAB_2_P project.

\section{Narrow Sense Heritability of Secondary Metabolites in Echinacea Angustifolia Kenan Touma', Rui Liu', Phil Hintz², John T. Arnason'1, Cory S. Harris' \\ ${ }^{1}$ Department of Biology, University of Ottawa, Canada \\ ${ }^{2}$ Trout Lake Farm, USA}

Echinacea has long been used for many medicinal properties such as anti-inflammatory and anti-microbial effects. Some of the most prominent secondary metabolites in echinacea are caffeic acid derivatives (CADs) including caftaric acid, chlorogenic acid, cichoric acid, and echinacoside and numerous alkylamides, which have a similar structure containing a polyunsaturated aliphatic fatty acid chain and a shorter substituent at the amine. Both AKAs and CADs are 
promising bioactive compounds that exert a variety of biological-pharmacological effects. With the overall objective of developing varieties of echinacea with enhanced phytochemical profiles, we completed preliminary breeding trials to assess the heritability of AKA and CAD production. To do so, we sampled the roots of 50 individuals of E. angustifolia from a genetically diverse population, cloning each plant by root propagation in the process. Hydroethanolic root extracts were analysed by HPLC-DAD and individuals with high or low CADs or AKAs were grouped in isolated breeding plots. Seeds were collected and planted the following spring. AKA and CAD levels in root samples of F1 generation plants were then analyzed and compared to the F0 generation and corresponding parent plants to calculate narrow-sense heritability. Our findings demonstrate that production of selective breeding based on either CADs or AKAs levels can enhance or diminish production in a single generation, but often at the expense of non-targeted metabolites. While additional research is required to confirm the heritability of these phytochemical traits, our results provide proof of principle and an important step toward "elite" Echinacea varieties and their products.

\section{Conflicts of Interest}

The authors declare that they have no conflicts of interest.

\section{Authors' Contributions}

CSH: Chair of the Program and Local Planning committee, made the call for abstracts on behalf of the NHPRS, reviewed the abstracts for acceptance as "live" and "contributed" presentations, and contributed to the copyediting and review of the publication.

(iD) http://orcid.org/0000-0002-0657-1838

JTA: Chair of the Program and Local Planning committee, made the call for abstracts on behalf of the NHPRS, reviewed the abstracts for acceptance as "live" and "contributed" presentations, and contributed to the copyediting and review of the publication.

(D) https://orcid.org/0000-0001-5354-5715

$\mathrm{BH}$ : Chair of the Program and Local Planning committee, made the call for abstracts on behalf of the NHPRS, reviewed the abstracts for acceptance as "live" and "contributed" presentations, and contributed to the copyediting and review of the publication.

PSH: Member of the Program and/or Local Planning committee, and reviewed abstracts for acceptance as "live" and "contributed" presentations.

(D) https://orcid.org/0000-0002-8782-5165

RMG: Member of the Program and/or Local Planning committee, and reviewed abstracts for acceptance as "live" and "contributed" presentations.

(D) https://orcid.org/0000-0002-0251-7025

BC: Member of the Program and/or Local Planning committee, and reviewed abstracts for acceptance as "live" and "contributed" presentations.

MJS: Member of the Program and/or Local Planning committee, and reviewed abstracts for acceptance as "live" and "contributed" presentations.

SS: Member of the Program and/or Local Planning committee, and reviewed abstracts for acceptance as "live" and "contributed" presentations.

PO: Member of the Program and/or Local Planning committee, and reviewed abstracts for acceptance as "live" and "contributed" presentations.

HQ: Member of the Program and/or Local Planning committee, and reviewed abstracts for acceptance as "live" and "contributed" presentations.

(D) https://orcid.org/0000-0002-7879-3093

JYN: Member of the Program and/or Local Planning committee, reviewed abstracts for acceptance as "live" and "contributed" presentations, and contributed to the copyediting and review of the conference abstract book.

(D) https://orcid.org/0000-0003-0031-5873 


\section{Funding}

Funding was generously provided by sponsors: Isura, Genuine Health, PGBBiopharma, Abbey Island Foods, Souroubea Botanicals and PEl Bioalliance.

\section{Article Information}

Managing Editor: Kieran Cooley, Pierre Haddad

Peer Reviewers: This article was peer-reviewed by the NHPRS conference planning committee.

Article Dates: Published Jun 0621

\section{Citation}

Please cite this article as follows:

Harris CS, Arnason JT, Hall B, Haddad PS, Golsteyn RM, Chapman B, Smith MJ, Sidhu S, Ovadje P, Quach H, Ng JY. Canada and the Changing Global NHP Landscape: The 17th Annual Conference of the Natural Health Products Research Society of Canada. Journal of Natural Health Product Research. 2021 Jun 06: 3(1). https://jnhpresearch. com/index.php/jnhpr/article/view/20

DOI Link: https://doi.org/10.33211/jnhpr.20

\section{Copyright}

(c) Cory S. Harris, John T. Arnason, Braydon Hall, Pierre S. Haddad, Roy M. Golsteyn, Bob Chapman, Michael J. Smith, Sharan Sidhu, Pamela Ovadje, Halton Quach, Jeremy Y. Ng. (2021). Published first in the Journal of Natural Health Product Research. This is an open access article distributed under the terms of the Creative Commons Attribution License (https://creativecommons.org/licenses/by/4.0/), which permits unrestricted use, distribution, and reproduction in any medium, provided the original work, first published in the Journal of Natural Health Product Research, an NHP Publications journal, is properly cited. The complete bibliographic information, a link to the original publication on https://www.jnhpresearch.com, as well as this copyright and license information must be included.
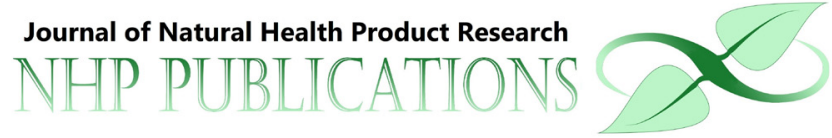

Canadà̀

Does the safety, efficacy, and quality of natural health products matter to YOU?

Submit your research article to the Journal of Natural Health Product Research!

Pre-submission inquiries? Send us an email at editorial.office@jnhpresearch.com Facebook, Twitter and LinkedIn: @NHPPublications 